\title{
Basal Ganglia Output Has a Permissive Non-Driving Role in a Signaled Locomotor Action Mediated by the Midbrain
}

\author{
Sebastian Hormigo, Ji Zhou, Dorian Chabbert, Bharanidharan Shanmugasundaram, and \\ (1) Manuel A. Castro-Alamancos \\ Department of Neuroscience, University of Connecticut School of Medicine, Farmington, Connecticut 06001
}

The basal ganglia are important for movement and reinforcement learning. Using mice of either sex, we found that the main basal ganglia GABAergic output in the midbrain, the substantia nigra pars reticulata ( $\mathrm{SNr}$ ), shows movement-related neural activity during the expression of a negatively reinforced signaled locomotor action known as signaled active avoidance; this action involves mice moving away during a warning signal to avoid a threat. In particular, many SNr neurons deactivate during active avoidance responses. However, whether $\mathrm{SNr}$ deactivation has an essential role driving or regulating active avoidance responses is unknown. We found that optogenetic excitation of $\mathrm{SNr}$ or striatal GABAergic fibers that project to an area in the pedunculopontine tegmentum (PPT) within the midbrain locomotor region abolishes signaled active avoidance responses, while optogenetic inhibition of $\mathrm{SNr}$ cells (mimicking the $\mathrm{SNr}$ deactivation observed during an active avoidance behavior) serves as an effective conditioned stimulus signal to drive avoidance responses by disinhibiting PPT neurons. However, preclusion of $\mathrm{SNr}$ deactivation, or direct inhibition of $\mathrm{SNr}$ fibers in the PPT, does not impair the expression of signaled active avoidance, indicating that $\mathrm{SNr}$ output does not drive the expression of a signaled locomotor action mediated by the midbrain. Consistent with a permissive regulatory role, $\mathrm{SNr}$ output provides information about the state of the ongoing action to downstream structures that mediate the action.

Key words: avoidance; accumbens; escape; movement; striatum; threat

Significance Statement

During signaled active avoidance behavior, subjects move away to avoid a threat when directed by an innocuous sensory stimulus. Excitation of GABAergic cells in the substantia nigra pars reticulata ( $\mathrm{SNr}$ ), the main output of the basal ganglia, blocks signaled active avoidance, while inhibition of $\mathrm{SNr}$ cells is an effective stimulus to drive active avoidance. Interestingly, many $\mathrm{SNr}$ cells inhibit their firing during active avoidance responses, suggesting that $\mathrm{SNr}$ inhibition could be driving avoidance responses by disinhibiting downstream areas. However, interfering with the modulation of $\mathrm{SNr}$ cells does not impair the behavior. Thus, $\mathrm{SNr}$ may regulate the active avoidance movement in downstream areas that mediate the behavior, but does not drive it.

\section{Introduction}

The basal ganglia control movement and are involved in action selection and reinforcement learning (Schultz, 1998; Redgrave et al., 1999; Hikosaka et al., 2000; Klaus et al., 2019). Its critical role in movement becomes obvious during pathology, such as in Parkinson's disease, when basal ganglia output is abnormal and

\footnotetext{
Received May 4, 2020; revised Nov. 25, 2020; accepted Dec. 2, 2020.

Author contributions: S.H., J.Z., D.C., B.S., and M.A.C.-A. performed research; S.H., J.Z., D.C., B.S., and M.A.C.-A. analyzed data; S.H., J.Z., D.C., B.S., and M.A.C.-A. edited the paper; M.A.C.-A. designed research; M.A.C.-A. wrote the first draft of the paper; M.A.C.-A. wrote the paper.

The authors declare no competing financial interests.

This work was supported by National Institutes of Health grants to M.A.C.-A. We thank the National Institute on Drug Abuse drug supply program for providing clozapine-N-oxide (additional information at www.castro-lab.org). We thank Drs. Christopher Bonin and Geneva Hargis for help with manuscript editing.

Correspondence should be addressed to Manuel A. Castro-Alamancos at mcastro@uchc.edu.

https://doi.org/10.1523/JNEUROSCI.1067-20.2020

Copyright $\odot 2021$ the authors
}

movement is impaired (Bevan et al., 2002; Kravitz et al., 2010; Wichmann and Dostrovsky, 2011; Rubin et al., 2012; Willard et al., 2019). However, inactivation of basal ganglia output nuclei in primates has only subtle effects on motor behavior, mostly affecting movement vigor (Desmurget and Turner, 2008; Turner and Desmurget, 2010). The substantia nigra pars reticulata $(\mathrm{SNr})$ is the main output of the basal ganglia in the midbrain; SNr neurons are GABAergic, normally tonically active, and can pause their tonic firing during orienting movements (Hikosaka and Wurtz, 1983; Wurtz and Hikosaka, 1986; Chevalier and Deniau, 1990). A leading view is that, during action selection, the striatum integrates cortical and thalamic inputs that deactivate $\mathrm{SNr}$ neurons through the direct striatonigral pathway. In turn, $\mathrm{SNr}$ deactivation disinhibits its targets to drive appropriate movements for a specific context (in the present paper, drive is used as synonym of generate, trigger, and cause). Simultaneously, the indirect striatonigral pathway activates other $\mathrm{SNr}$ neurons 
inhibiting unwanted actions (Albin et al., 1989; DeLong, 1990; Mink, 1996; Hikosaka et al., 2000). Direct and indirect striatal neurons are usually silent in the absence of movement but activate similarly during movement (Cui et al., 2013; Tecuapetla et al., 2014; Barbera et al., 2016), continuously coding movement variables, such as speed (Markowitz et al., 2018; Sales-Carbonell et al., 2018), likely driven by their sensitivity to somatosensory input (Carelli and West, 1991; Robbe, 2018). Tonically active $\mathrm{SNr}$ neurons can either activate further or deactivate during movement (Gulley et al., 1999; Fan et al., 2012; Pan et al., 2013; Schmidt et al., 2013) and, like its afferent striatal neurons, continuously code movement variables (Barter et al., 2015; Yin, 2017). While it is evident that normal basal ganglia output codes movement variables and that abnormal output leads to severe movement disorders, it is not clear whether the normal neural signals output by the basal ganglia are critically required to drive (i.e., are necessary to generate) appropriate movements in specific behavioral contexts, such as during learned conditioned responses. Here we explored the role of basal ganglia output, via $\mathrm{SNr}$, on a sensory-guided locomotor conditioned behavior, known as signaled active avoidance.

Animals, including humans, readily learn to avoid harmful and threatening situations by moving in response to cues (e.g., fire alarm, traffic light). During signaled active avoidance, animals avoid a harmful unconditioned stimulus (US) by moving away (e.g., shuttling in a cage) during an interval signaled by a conditioned stimulus (CS) (Mowrer, 1960; Bolles, 1970; Mineka, 1979; LeDoux et al., 2017). Midbrain circuits are capable of mediating signaled active avoidance (Cohen and Castro-Alamancos, 2007, 2010a,b). Furthermore, manipulation of SNr activity can fully control active avoidance responses (Hormigo et al., 2016). $\mathrm{SNr}$ activation blocks avoidance responses by inhibiting cells in the midbrain pedunculopontine tegmentum (PPT), a main part of the midbrain locomotor region that is critical for signaled active avoidance expression (Hormigo et al., 2019), whereas SNr deactivation drives avoidance responses in the absence of any external sensory stimulus. However, the role of $\mathrm{SNr}$ in mediating signaled active avoidance in response to a sensory stimulus is unclear. It may be critically involved in driving avoidance responses by disinhibiting PPT neurons or, alternatively, may have a permissive gating role. In a permissive gating role, $\mathrm{SNr}$ deactivation would simply allow expression of active avoidance responses, whereas excessive $\mathrm{SNr}$ activation would impede them; that is, the overall SNr tonic firing mode/state would gate (allow) avoidance responses, while discrete SNr firing would monitor (signal) avoidance movement, but not drive (cause) it. Using a combination of electrophysiology, optogenetics, and chemogenetics (designer receptor exclusively activated by designer drugs [DREADDs]) in behaving mice, we found that SNr neurons are robustly modulated during movements related to signaled active avoidance, but this activity is not critically required for the expression of the behavior.

\section{Materials and Methods}

Experimental design and statistical analysis. All procedures were reviewed and approved by the Animal Care Committee of Drexel University and conducted in adult ( $>8$ weeks) male and female mice. The results from both sexes were combined since there is no sex difference in the behavior measured for the strains used (Hormigo et al., 2019).

All experiments involved a repeated-measures design in which the mice or cells serve as their own controls. Unless otherwise stated, conclusions are based on within-subject comparisons. We tested for a main effect (e.g., light) using a two-way mixed-design ANOVA followed by comparisons with Tukey's test. In the mixed-design ANOVA, the repeated-measures factor was the light effect (with as many levels as conditions tested) and the other factor was the animals' sessions (behavior) or the repetition of the same protocols (cells). The sessions factor is statistically independent because the fully trained animals must perform the control signaled active avoidance trials (auditory CS [ACS] trials) at the same high levels per session (by definition one session has no effect on task performance on the other sessions). Tukey tests were conducted for the repeated-measures factor when the within-subjects effect ( $F$ value) was statistically significant at a level of $p<0.01$. Behavioral optogenetic experiments consist of a balanced design wherein the tested light (e.g., light CS [(LCS] trials) and control conditions (e.g., ACS trials) are randomly distributed within the same session, which is repeated on multiple days. Thus, all comparisons are between conditions presented within the same session. We highlight results with significance levels of $p<0.01$. To test for equivalency between ACS and LCS alone trials, we first used the mixed ANOVA and Tukey's to establish that the trials were not significantly different $(p>0.01)$. This was followed by an equivalence paired test in which the upper boundary for equivalence was set at $20 \%$. A power analysis was conducted with OriginLab Pro using the measured means difference variability. It revealed that 3 animals in which we conducted 5 identical daily sessions per animal ( 15 sessions) was sufficient to detect a $\sim 20 \%$ change in avoidance rate with a power of 0.99 $(p<0.05)$. This was used as the bare minimum number of animals and sessions per group.

The experiment timeline included the following sequence of phases: adeno-associated viral (AAV) vector injections (unless mice natively expressed opsins), active avoidance learning phase, optical fiber implantation, active avoidance testing phase, and in vivo/in vitro recordings (some cases) followed by histology. In naive mice, the active avoidance learning phase occurred after optical fiber implantation. In mice subjected to AAV injections (optogenetics or DREADDs), the active avoidance testing phase commenced 3-5 weeks after AAV injections. In mice subjected to optogenetics, optical fiber implantation occurred 5-6d before commencing the active avoidance testing phase, which lasted 35 weeks with daily (weekday) sessions.

To enable rigorous approaches, we maintain a local server with a central database accessed through a wiki that logs all details and metadata related to the experiments, including all information about animals and details about surgical procedures, behavioral sessions, electrophysiological recordings, histology, and scripts used for analyses. Moreover, during daily behavioral sessions or electrophysiological recordings, computers run experiments automatically using preset parameters logged for reference during analysis. Analyses are performed using scripts that automate all aspects of data analysis from access to metadata and data files to population statistics and graph generation (scripts and metadata will be accessible through our website or by request).

Strains and AAVs. The following AAVs (injected undiluted) and mouse strains were used in the present study. To inhibit SNr GABAergic cells using optogenetics, we expressed Arch using two different approaches. In the Vgat-SNr-Arch group, we injected Vgat-cre mice (Jax 028862; B6J.129S6(FVB)-Slc32a $1^{\text {tm2(cre)Lowl }}$ MwarJ) with AAV5-EF1aDIO-eArch3.0-EYFP (UNC Vector Core, titers: $3.4 \times 10^{12} \mathrm{GC} / \mathrm{ml}$ by dot blot) to express eArch3.0 in SNr GABAergic cells. In the Vgat-Arch group, we crossed Vgat-cre and Ai40D (Jax 021188; B6.Cg-Gt(ROSA) 26Sor $\left.{ }^{\text {tm } 40.1(C A G-a o p 3 / E G F P) H z e} / J\right)$ mice to express ArchT in GABAergic cells. To excite striatal GABAergic fibers using optogenetics, we injected Vgat-cre mice with AAV5-EF1a-DIO-hChR2(H134R)-eYFP (UPenn Vector Core, titers: $1.8 \times 10^{13} \mathrm{GC} / \mathrm{ml}$ by qPCR) to express ChR2 in striatum cells. As a No-Opsin AAV control, we used AAV8-hSyn-EGFP (Addgene, titers: $4.3 \times 10^{12} \mathrm{GC} / \mathrm{ml}$ by qPCR). To excite GABAergic cells using DREADDs, we injected Vgat-cre mice with AAV5-hSyn-DIOhM3D(Gq)-mCherry (UNC Vector Core, titers: $3.8 \times 10^{12} \mathrm{GC} / \mathrm{ml}$ by dot blot). To inhibit GABAergic cells using DREADDs, we injected Vgat-cre mice with AAV5-hSyn-DIO-hM4D(Gi)-mCherry (UNC Vector Core, titers: $5.4 \times 10^{12} \mathrm{GC} / \mathrm{ml}$ by dot blot).

Surgeries. Optogenetics experiments involved bilaterally injecting $0.3 \mu \mathrm{l}$ AAVs per site during isoflurane anesthesia $(\sim 1 \%)$. Animals 
received carprofen after surgery. The stereotaxic coordinates for injection sites (in millimeters from bregma; lateral from the midline; ventral from the bregma- $\lambda$ plane) are as follows: $\mathrm{SNr}$ (3.3 posterior; $1.4-1.5 ; 4$ $4.1)$, dorsal striatum $(\mathrm{StrD})(1.1$ anterior; $1.8 ; 2.5)$, ventral striatum (StrV) (1.1 anterior; $1.8 ; 4)$, and NAc (Acb) (1.2 anterior; $0.8 ; 4.3)$. The coordinate ranges reflect different animals that were grouped together because the slight coordinate differences produced similar effects.

In optogenetics experiments, a dual optical fiber $(200 \mu \mathrm{m}$ in diameter) was implanted bilaterally during isoflurane anesthesia at the abovementioned coordinates and held in place with a combination of screws, cyanoacrylate, and dental cement. Bilateral cannulas were implanted in the injection site or in the projection site of the AAV injection site. The stereotaxic coordinates for the implanted cannulas (in $\mathrm{mm}$ ) are: $\mathrm{SNr}(3.3$ posterior; $1.5 ; 4.1)$ and PPT (4.7 posterior; 1.25 ; 3.1 entering in the posterior direction at a $20^{\circ}$ angle).

Optogenetics. The implanted dual optical fibers were connected to patch cables using sleeves. A black aluminum cap covered the head implant and completely blocked any light exiting at the ferrule junction. Furthermore, the experiments occurred in a brightly lit cage that made it difficult to detect any light escaping the implant. The other end of the patch cables was connected to a dual light swivel (Doric Lenses) that was coupled to a blue laser $(450 \mathrm{~nm} ; 80 \mathrm{~mW})$ or a green laser $(520 \mathrm{~nm} ; 100$ $\mathrm{mW})$.

Optogenetic sessions use either blue or green light to activate ChR2 and Arch, respectively. The electrophysiological effects of optogenetic blue light patterns (continuous and $2-100 \mathrm{~Hz}$ trains of $1 \mathrm{~ms}$ pulses) and green light (continuous) were previously characterized using both whole-cell recordings in slices and single-unit recordings in anesthetized mice in vivo (Hormigo et al., 2016, 2019). We found that there were varying electrophysiological and behavioral effects from different patterns of blue light, so during behavior it is important to test the effects of continuous pulses and different frequency trains of blue light. Therefore, we also performed electrophysiology validation experiments, using the previously described methods, in most of the animals from this study (the validation data presented in the present study correspond to animals from this study). We found that continuous green or blue light robustly inhibited SNr cells that express eArch3.0/ArchT or IC ++ , respectively, both in slices and in isoflurane-anesthetized mice in vivo (see Fig. 4A-E). We also tested the effects of blue light applied to fibers of ChR2-expressing GABAergic cells at their postsynaptic targets (see Fig. 9A-C).

The blue light stimuli used during optogenetics sessions to activate ChR2 included 8 different patterns of light delivered randomly at the same power consisting of continuous pulses and trains of $1 \mathrm{~ms}$ pulses at $2,5,10,20,40,66$, or $100 \mathrm{~Hz}$. On the first optogenetics session, we tested different blue light powers up to a maximum of $\sim 3 \mathrm{~mW}$ and selected the maximum power that did not interfere with the ability of the animal to escape the US during any of the light patterns used. In most cases, when the regular maximum blue light power did not interfere with the ability of the animal to avoid or escape the US, we conducted additional sessions using a higher blue light power up to a maximum of $6 \mathrm{~mW}$. The green light used during optogenetics sessions to activate Arch was always continuous and was tested at one power $(25-35 \mathrm{~mW})$ within a session or at different powers $(3,15,25,35$, or $45 \mathrm{~mW})$ randomly delivered within a session. Unless otherwise noted, the behavioral experiments used green light between 25 and $35 \mathrm{~mW}$ and blue light between 0.4 and $6 \mathrm{~mW}$. Power is regularly measured by flashing the connecting patch cords onto a light sensor with the sleeve on the ferrule.

Active avoidance task in a shuttle box. Mice were trained in the active avoidance task using procedures similar to those described previously for rats and mice (Cohen and Castro-Alamancos, 2007, 2010b; Hormigo et al., 2016, 2019). In most experiments, the mice were trained before reaching the testing phase; learning occurred during 4-6 daily sessions (50 trials per session) after AAV injections and before cannula implantation (see timeline above). In experiments in which we studied naive animals, active avoidance learning occurred during the testing phase. All reported active avoidance data were gathered during the testing phase.

During an active avoidance session, mice are placed in a standard shuttle box $(16.1$ inch $\times 6.5$ inch $)$ that has two compartments separated by a partition with side walls forming a doorway that the animal has to traverse to shuttle between compartments. For active avoidance training during the learning phase, a trial consists of a $7 \mathrm{~s}$ avoidance interval followed by a $10 \mathrm{~s}$ escape interval (see Fig. 1). During the avoidance inter$\mathrm{val}$, an ACS is presented for the duration of the interval or until the animal produces a conditioned response (avoidance response) by moving to the adjacent compartment, whichever occurs first. If the animal avoids by moving to the next compartment, the CS ends, the escape interval is not presented, and the trial terminates. However, if the animal does not avoid, the escape interval ensues by implementing white noise and a mild scrambled electric foot-shock $(0.3 \mathrm{~mA})$ delivered through the grid floor of the occupied half of the shuttle box. This US readily drives the animal to move to the adjacent compartment (escape response), at which point the US terminates, and the escape interval and the trial ends. Thus, an avoidance response is a response that will eliminate the imminent presentation of a harmful stimulus that the animal will readily escape if presented. An escape response is the response driven by presentation of the harmful stimulus to eliminate the harm it causes. In principle, successful avoidance is highly adaptive because it warrants the absence of harm. Each trial is followed by an intertrial interval (duration is randomly distributed; $25-45 \mathrm{~s}$ range), during which the animal awaits the next trial.

Active avoidance trial types. Within daily sessions, we tested different types of trials (see schematic diagrams in Figs. 1A, 10A) presented randomly within the active avoidance task. In ACS trials, an ACS $(8 \mathrm{kHz}$ tone at $\sim 85 \mathrm{~dB}$ ) signals the avoidance interval (see Fig. $1 A$ ). ACS trials are control trials that represent the normal performance of the animal during a session. Every block of 10 trials within an optogenetics session has 5 randomly presented ACS trials to assure that the animal continues to perform the task in the absence of the optogenetic manipulation. In No CS trials, the avoidance interval is not signaled by any stimulus and the escape interval does not occur (see Fig. 1A). The purpose of No CS trials is to determine the percentage of avoidance responses performed by chance because of motor activity. In No US trials, a CS occurs during the avoidance interval, but the escape interval is not presented; there is no consequence for the CS. The purpose of No US trials is to determine whether the CS produces an innate response (e.g., shuttling) in untrained animals or whether it is a true neutral stimulus before training. In addition to ACS trials, No CS trials, or No US trials, optogenetics sessions contain one or more of the following trials: LCS alone trials, ACS + LCS trials, LS-ACS trials, and LS trials.

In LCS alone trials, the avoidance interval is signaled only by light delivered into the brain (see Fig. $1 A$ ). The purpose of LCS alone optogenetic sessions is to compare LCS alone trials versus ACS trials to determine whether the LCS is capable of driving avoidance responses as effectively as the ACS. Thus, in LCS alone sessions, we indicate nonsignificant differences $(p>0.01)$ between LCS alone trials and ACS trials to highlight optogenetic stimuli that were equivalent to the ACS in driving avoidance responses.

In ACS + LCS trials, the avoidance interval is signaled by the same ACS as in ACS trials and optogenetic light is simultaneously delivered into the brain (see Fig. 1A). The purpose of ACS+LCS optogenetic sessions is to compare ACS + LCS trials versus ACS trials to determine whether the LCS has any effect on avoidance responses driven by the ACS. In ACS+LCS or LCS alone trials, the light persists during the escape interval but not during the intertrial interval. Thus, the optogenetic light delivered per trial depends on the duration of the avoidance (maximum $7 \mathrm{~s}$ ) and escape intervals ( $<3 \mathrm{~s}$ for all mice), which the animals control.

In LS-ACS trials, the trials are identical to those of ACS + LCS trials, but optogenetic green light begins randomly at between one-half and three-fourths of the intertrial interval preceding the avoidance interval and continues during the avoidance and escape intervals until the animal avoids or escapes (see Fig. 10A). This typically results in $\sim 10 \mathrm{~s}$ of green light per trial $(<15 \mathrm{~s})$. Intertrial crossings have no effect on the optogenetic light presentation. The purpose of LS-ACS trials is to determine whether inhibiting SNr firing starting during the intertrial interval has any effect on avoidance responses driven by the upcoming ACS trial. The inhibition precludes the normal modulation of $\mathrm{SNr}$ activity that 
occurs during the avoidance interval as the animal avoids in response to the ACS.

In LS trials, everything is identical to the LS-ACS trials, but the ACS is not presented during the avoidance interval and the escape interval does not exist (see Fig. $10 A$ ). The purpose of LS trials is to serve as controls for LS-ACS trials by determining the probability that the animal will shuttle during the avoidance interval in the presence of the optogenetic LS stimulation alone (without the ACS).

All optogenetics experiments involved a repeatedmeasures design in which different randomly presented trials are compared within the same session (50-150 trials per session). Half of the trials in a session are control trials (e.g., ACS trials), and the other half are optogenetics trials (e.g., ACS+LCS or LCS alone trials) that used different light stimuli. Statistical comparisons consist of repeated measures between different trial types tested within a session, and significant statistical differences versus ACS trials $(\alpha=0.01)$ are indicated in each figure.

Active avoidance task variations. We used two different avoidance tasks (AA1 and AA2) based on the consequence of intertrial crossings during the intertrial interval. In AA1, mice were free to cross between compartments, and there was no consequence for intertrial crossings. AA1 is the usual format of the active avoidance task, but mice may increase the number of spontaneous crossings (shuttling) causing spurious avoidance responses. To control this possibility, we measure the number of spurious avoidance responses by presenting unsignaled avoidance intervals that have no consequence (i.e., NoCS trials) and we use the AA2 task. In AA2, mice receive a $0.2 \mathrm{~s}$ footshock $(0.3 \mathrm{~mA})$ and white noise for each intertrial crossing. Therefore, in AA2, mice must passively avoid during the intertrial interval, by inhibiting their tendency to shuttle between trials, and actively avoid during the signaled avoidance interval. Thus, AA2 tests signaled active avoidance during the signaled avoidance interval and unsignaled passive avoidance during the unsignaled intertrial interval. In addition, we used a CS discrimination task (AA3) in which mice must respond differently to a CS1 and a CS2 presented randomly (see Fig. $5 A$ ). In AA3, animals perform regular signaled active avoidance to CS1, and perform signaled passive avoidance to CS2; intertrial crossings are not punished. If animals shuttle during the CS2 avoidance interval, they receive a $0.5 \mathrm{~s}$ foot-shock $(0.3 \mathrm{~mA})$ with white noise and the trial ends. If animals do not shuttle during the CS2 avoidance interval, the CS2 trial terminates at the end of the avoidance interval. While AA1, AA2, and AA3 test signaled active avoidance, AA2 also tests unsignaled passive avoidance during the intertrial interval, and AA3 also tests signaled passive avoidance during CS2. Furthermore, both AA2 and AA3 minimize or eliminate the number of spurious avoidance responses.

Behavioral measures and video tracking in the shuttle box. There are three main variables representing task performance in the shuttle box. The percentage of active avoidance responses (\% avoids) represents the trials in which the animal actively avoided the US in response to the CS. The response latency (latency) represents the time (seconds) at which the animal enters the safe compartment after the CS onset. The number of crossings during the intertrial interval (intertrial crossings) represents either random shuttling because of locomotor activity in the AA1 and AA3 procedures or failures to passively avoid in the AA2 procedure.

Animals are also video tracked (30 FPS) during active avoidance sessions that used optogenetics. The tracking followed color markers

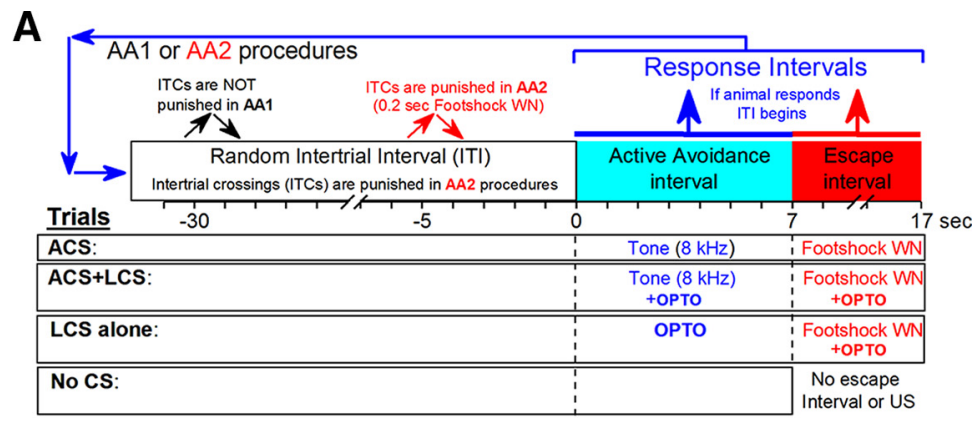

B Active avoidance in Shuttle Box (ACS trials, AA1 procedure)
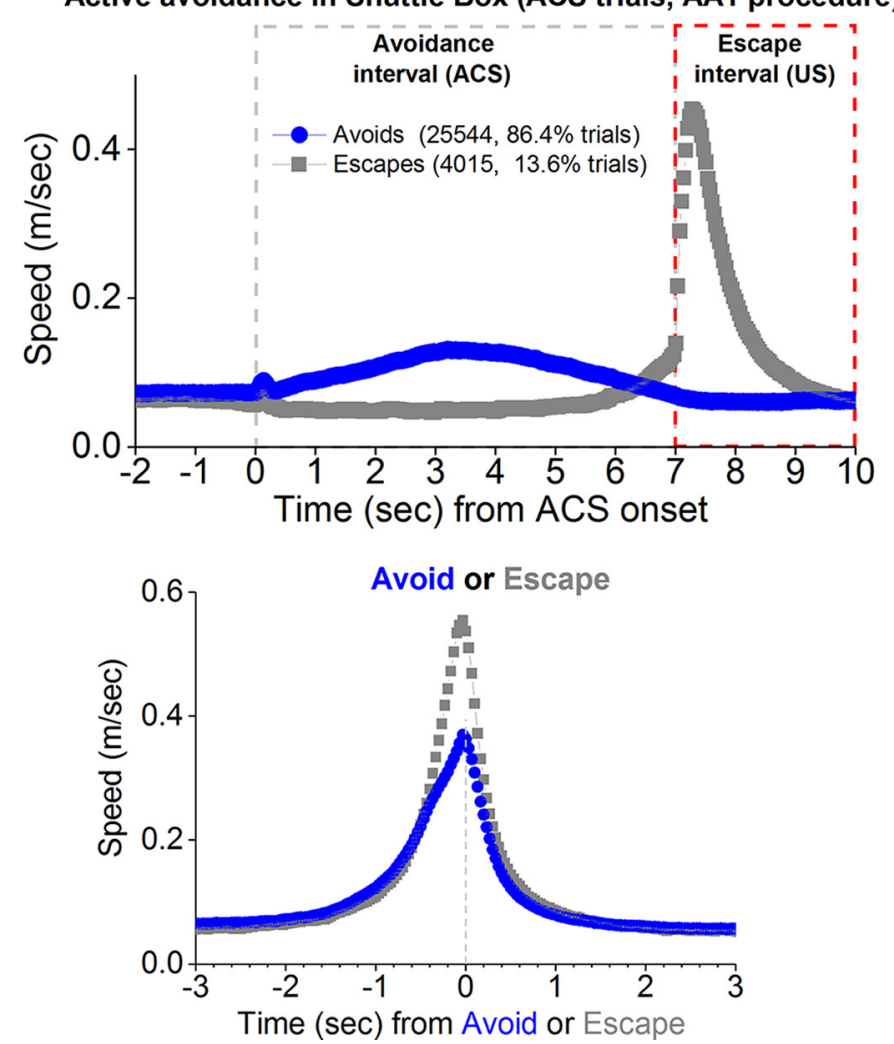

Figure 1. Basic active avoidance trials. $A$, Schematic of the basic types of trials (ACS, ACS $+L C S$, LCS alone, and No (S trials) used in this study. Active avoidance trials consist of a sequence of avoidance, escape, and intertrial intervals. An effective response during the avoidance interval (e.g., shuttling in a shuttle box) avoids the occurrence of the escape interval (foot-shock and white noise [WN]). The stimulus that signals the active avoidance interval defines each trial type. In ACS trials, an $8 \mathrm{kHz}$ auditory tone (ACS) signals the active avoidance interval. In LCS alone trials, optogenetic (OPTO) light (LCS) signals the active avoidance interval. In ACS + LCS trials, the ACS and LCS are delivered simultaneously during the avoidance interval to determine whether the LCS affects the ability of the ACS to drive avoidance responses. In No CS trials, the avoidance interval is not signaled to measure the incidence of spurious avoidance responses because of exploratory movement. When the optogenetic light is delivered during the avoidance interval, it continues during the escape interval until the animal escapes. The difference between AA1 and AA2 procedures is that in AA2 the intertrial crossings (ITCS) that occur during the intertrial interval (ITI) are punished ( $0.2 \mathrm{~s}$ foot-shock and WN). Thus, in AA2, the intertrial interval is a passive avoidance interval. $\boldsymbol{B}$, Video tracking showing movement speed from a group of mice $(n=27$ from this study) performing ACS trials in a shuttle box. Top, The instantaneous speed ( \pm SEM) is plotted versus the onset of ACS trials for avoidance responses (avoids; blue) and escape responses (escapes; gray). Bottom, The instantaneous speed ( \pm SEM) is plotted versus the occurrence of avoidance or escape responses revealing the faster speed of escapes.

located on the head connector above the nose and between the ears. Several movement (tracking) measures were derived during active avoidance. Distance was the number of pixels crossed by the animal in its trajectory during the avoidance and escape intervals of a trial (trial distance) or during the intertrial interval (intertrial distance). Trial speed was the trial distance divided by the response latency. Intertrial speed 
was the intertrial distance divided by the intertrial interval duration. Trial velocity was the displacement divided by the response latency (displacement was the number of pixels in a straight line between the position of the animal at trial start and the position of the animal at trial end when the animal avoided or escaped). Pixel measures were converted to metric units using calibrations. Trial speed and intertrial speed represent the overall movement of the animal in any direction during those periods, whereas trial velocity represents movement in the correct direction to avoid.

DREADDs. As a supplement to optogenetics experiments, we used DREADDs to inhibit GABAergic SNr cells, as in previous studies (Hormigo et al., 2016), and to excite or inhibit GABAergic striatum or NAc cells. In contrast to optogenetics, DREADDs modulate neural activity in a time-unselective manner. Mice were initially trained in 5 signaled active avoidance sessions (50 trials per daily session). Afterward, in successive daily sessions, animals were subjected to either saline or clozapine $\mathrm{N}$-oxide $(\mathrm{CNO} ; 20 \mathrm{mg} / \mathrm{kg}$, i.p.) injections $45 \mathrm{~min}$ before active avoidance performance (ACS trials). A series of (4 or 5) consecutive saline sessions were followed by a series of consecutive $\mathrm{CNO}$ sessions and this sequence was repeated 2 or 3 times.

Spherical treadmill SNr recordings during active avoidance. To record single-unit $\mathrm{SNr}$ activity during active avoidance, we tested active avoidance with head-fixed mice on a spherical treadmill. Mice underwent surgery (as described above) to implant a head-holder and create a craniotomy above SNr. The craniotomy was filled with silicone gel (Kwik-Sil), which was replaced during each recording session. Head-fixed mice were trained in an active avoidance task on a spherical treadmill $(20 \mathrm{~cm}$ ball). The position of the animal in virtual space was monitored with 2 ball sensors that provide movement in the horizontal and vertical planes ( $x, y$ coordinates). The system used a custom LabVIEW (National Instruments) program that controlled all task parameters.

On the spherical treadmill, mice were trained in ACS trials similar to those performed in a shuttle box (see Fig. 2). Each trial consisted of an avoidance interval ( $7 \mathrm{~s}$ ) followed by an escape interval (presented only if the animal does not avoid). An ACS $(8 \mathrm{kHz}$ tone at $\sim 85 \mathrm{~dB})$ signals the avoidance interval. The escape interval consists of white noise and a train of air-puffs ( $50 \mathrm{~ms}$ pulses at $10 \mathrm{~Hz} ; 30 \mathrm{PSI}$ ) delivered to the back/side of the mouse (US), which makes the animal run rapidly in the forward direction. To avoid or escape, the mouse is required to run $50 \mathrm{~cm}$ forward starting from the onset of the ACS. If this occurs during the avoidance interval, it counts as an avoidance response, the ACS turns off, and the escape interval does not occur. If the animal fails to avoid, the escape interval is presented until the animal reaches the $50 \mathrm{~cm}$ target (all mice escape successfully in $<5 \mathrm{~s}$ ). A random intertrial interval follows each trial, during which the animals are allowed to move freely. In trained mice, a sharp glass pipette (2-6 $\mathrm{M} \Omega$, filled with saline) was lowered into the $\mathrm{SNr}$ to record from well-isolated single units while performing active avoidance trials. Mice were recorded on multiple days in 2-3 h sessions (each unit was recorded for at least 60 trials). During the last recording session, a marking lesion was made at the top border of the $\mathrm{SNr}$ where neuronal sampling started.

Cross-correlations were calculated between the firing rate of the units and the speed using a $0.1 \mathrm{~s}$ bin during two periods of each trial; the avoidance interval and an equivalent period during the last half of the preceding intertrial interval. The avoidance interval period reflects avoidance movement and the intertrial interval reflects general movement.

Histology. Mice were deeply anesthetized with an overdose of ketamine. Upon losing all responsiveness to a strong tail pinch, the animal was decapitated and the brain was rapidly extracted and placed in fixative. The brain was sectioned ( $100 \mu \mathrm{m}$ sections) in the coronal or sagittal planes. Sections were mounted on slides, coverslipped with DAPI mounting media, and photographed using a fluorescent microscope. The location of the tips of the implanted cannulas was marked on 3D (coronal or sagittal) atlases generated in our laboratory. Thus, the sections containing the visible tracks of the cannula were matched with the $3 \mathrm{D}$ atlas sections and then the location of the track ending was marked, as described previously (Hormigo et al., 2019). In some cases, we reconstructed the result of AAV injections by tracing all the GFP fluorescence.

\section{Results}

\section{Summary of results}

First, we describe the basic signaled active avoidance trials used in the shuttle box procedure (see Fig. 1). Second, we describe a new signaled active avoidance procedure in head-fixed mice moving on a spherical treadmill that is based on the shuttle box procedure, but allows us to record $\mathrm{SNr}$ neurons as mice perform active avoidance. Using the spherical treadmill procedure, we found that many $\mathrm{SNr}$ neurons deactivate in relation to the avoidance movement (see Fig. 2). Third, to mimic the $\mathrm{SNr}$ deactivation observed during behavioral recordings, we used optogenetics (validated with electrophysiology; see Fig. 4A-E) to directly inhibit SNr neurons (in substitution of the CS presentation) during active avoidance in a shuttle box. We found that $\mathrm{SNr}$ inhibition is an effective CS to drive active or passive avoidance responses through learning (see Figs. 4F, 5). Fourth, since $\mathrm{SNr}$ neurons are normally deactivated by GABAergic inputs from the striatum, we tested the effect of optogenetically exciting GABAergic striatonigral fibers within $\mathrm{SNr}$ (originating in different portions of striatum) on active avoidance. The results show that, similar to direct inhibition of $\mathrm{SNr}$ cells, indirect inhibition of $\mathrm{SNr}$ cells by exciting GABAergic striatonigral afferents, is a highly effective CS to drive active avoidance responses (see Figs. 6, 7). We also found that excitation of GABAergic striatonigral fibers, particularly those originating in the NAc, can strongly suppress signaled active avoidance responses, but only when optogenetic trains at $\sim 40-66 \mathrm{~Hz}$ are used. This suppression of signaled active avoidance is because of the excitation of GABAergic fibers coursing through $\mathrm{SNr}$ from NAc to PPT, which very effectively inhibits PPT neurons at the noted optogenetic train frequencies (see Figs. 8, 9). These data provide more insight into our previous finding in which PPT was shown to be an essential hub for the expression of signaled active avoidance (Hormigo et al., 2019). Fifth, we tested the effect of optogenetically precluding $\mathrm{SNr}$ deactivation on signaled active avoidance by sustained inhibition of $\mathrm{SNr}$ cells that begins randomly during the intertrial interval preceding CS onset but found that this does not impair the active avoidance behavior (see Fig. 10). We reached a similar conclusion using DREADDs to manipulate the activity of striatal and $\mathrm{SNr}$ cells (see Fig. 11). Finally, we tested the effect of inhibiting GABAergic afferents reaching PPT from SNr (and zona incerta) on signaled active avoidance and found little impact on signaled active avoidance behavior (see Fig. 12). Together, the results indicate that the deactivation of $\mathrm{SNr}$ cells observed during signaled active avoidance is not driving the active avoidance response, but $\mathrm{SNr}$ activity can play a permissive, regulatory role.

\section{SNr cell activity during active avoidance responses}

A typical signaled active avoidance trial (ACS; Fig. $1 \mathrm{~A}$ ) in a shuttle box consists of consecutive avoidance (7 s signaled by an auditory tone; ACS) and escape (US foot-shock) intervals followed by a random intertrial interval (25-45 s). Mice readily learn to shuttle between compartments during the avoidance interval (avoidance response) to prevent the occurrence of the escape interval (Fig. $1 B$ ). However, recording single-unit $\mathrm{SNr}$ activity is challenging when the mice are free to move in a shuttle box.

To record from SNr single units, we used head-fixed mice moving on a spherical treadmill trained to perform a signaled active avoidance procedure (Fig. 2A). The contingencies on the spherical treadmill were similar to those on the shuttle box 
A

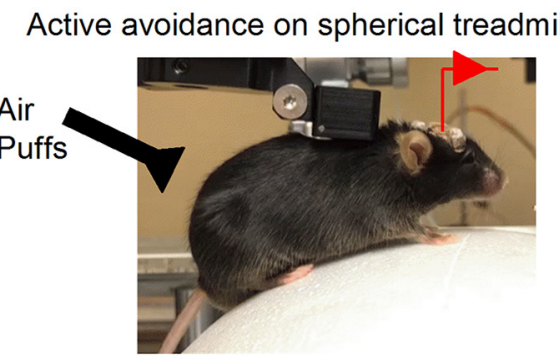

B

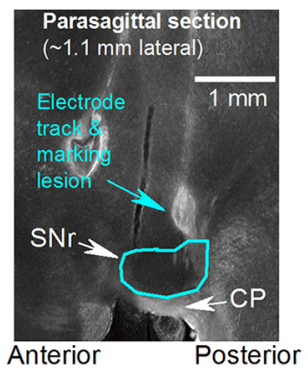

C

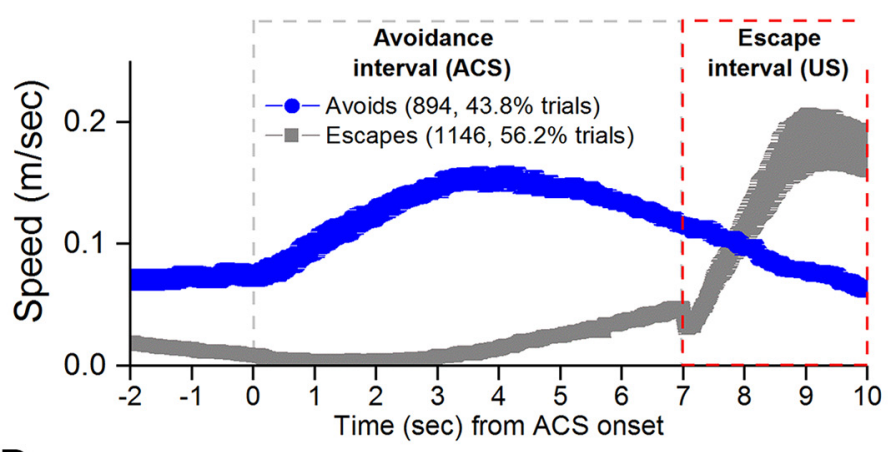

Avoid or Escape

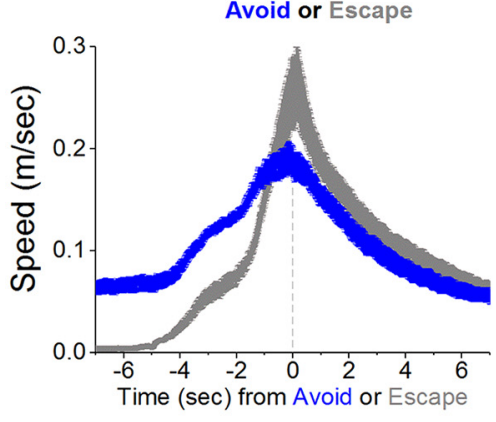

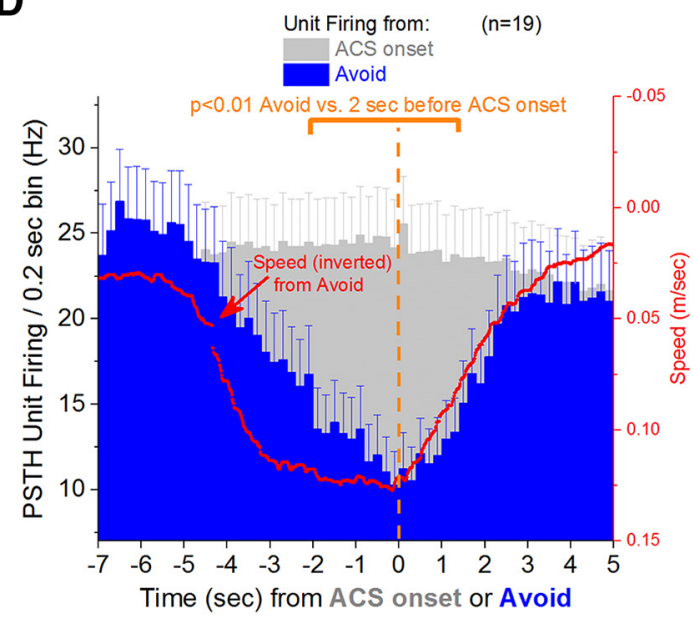

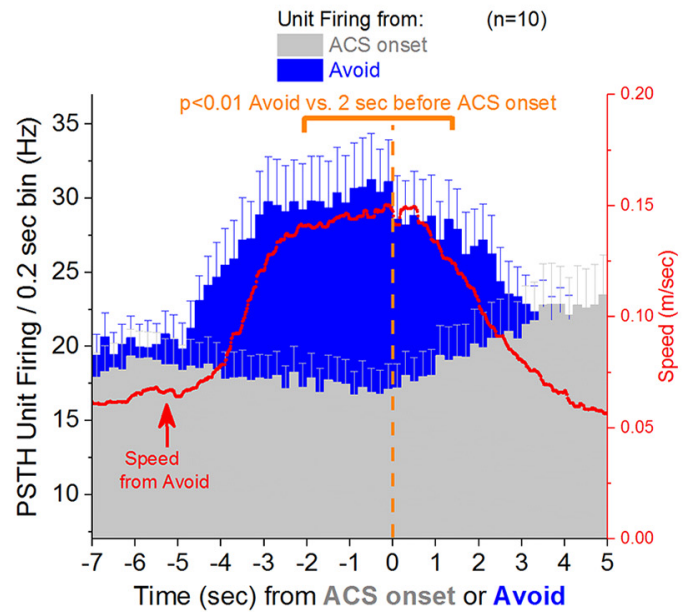

$\mathbf{E}$

Deactivated Units
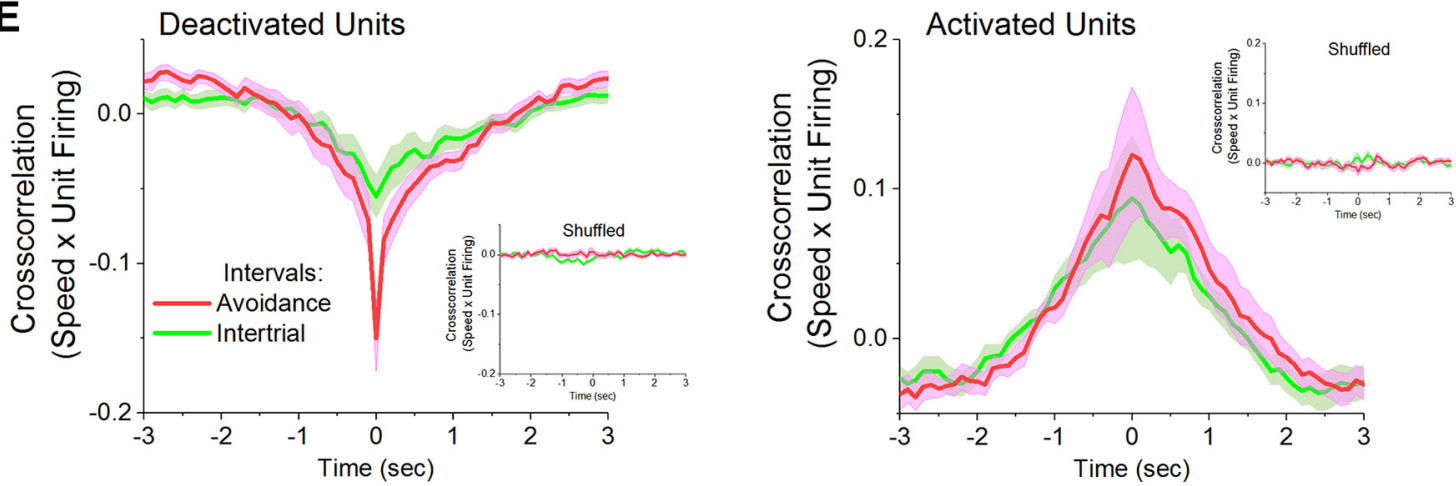

Figure 2. Active avoidance task in head-fixed mice on a spherical treadmill. A, Head-fixed mouse on the spherical treadmill used to record SNr cells during ACS trials. The mice are subjected to ACS trials detailed in Figure $1 A$, except that the US during the escape interval is a train of air puffs (delivered to the back of the animal) and white noise (WN); movement during the intertrial interval is not punished. $B$, Parasagittal section showing the location of an electrode marking lesion in the dorsal border of $\mathrm{SNr}$, at the point where the electrode enters the nucleus and cell sampling begins. $\mathrm{CP}$, Cerebral peduncle. C, Speed tracking during active avoidance performance obtained from a group of mice $(n=8)$ performing ACS trials on the spherical treadmill. Left, The instantaneous speed ( \pm SEM) is plotted versus the onset of ACS trials for avoidance responses (avoids; blue) and escape responses (escapes; gray). Right, The instantaneous speed ( \pm SEM) is plotted versus the occurrence of avoidance or escape responses. $\boldsymbol{D}$, Peristimulus time histograms (PSTHs) of SNr cells single-unit firing recorded during active avoidance performance on the spherical treadmill. Blue bars represent PSTHs where time 0 is the occurrence of the avoidance response. Gray bars represent PTSHs for the same data where time 0 is the onset of the ACS. The overlaid red lines plot the speed from the occurrence of the avoidance responses for the same trials. The plots represent two groups of cells based on their activity during active avoidance. Left, SNr cells $(n=19)$ that inhibited their firing (deactivated) during the occurrence of avoidance responses (note that speed is inverted for comparison). Right, SNr cells $(n=10)$ that enhanced their firing (activated) during the occurrence of avoidance responses. $\boldsymbol{E}$, Cross-correlations between movement speed and neuronal firing for the cells that deactivated and activated during the avoidance interval. The cross-correlations were calculated during the avoidance interval and during an equivalent period of the intertrial interval per every trial ( $0.1 \mathrm{~s} \mathrm{bin;}-3$ to $3 \mathrm{~s}$ ). For the shuffled cross-correlations (insets), one of the signals was randomly reordered before calculating the cross-correlation. 
procedure, except that the US during the escape interval was a train of air-puffs delivered to the back of the mouse (instead of a foot-shock), which makes the mouse run forward (escape) rapidly. To avoid presentation of the US, the mice had to move forward a defined distance $(50 \mathrm{~cm})$ during the ACS (avoidance interval). On the spherical treadmill, mice learned to avoid on about half of the trials (Fig. 2C). Typically, avoidance responses were likely when animals were active, as noted by the higher pretrial speed before successful avoidance trials (Avoids; Fig. 2C), compared with the almost nil pretrial speed of animals that failed to reach the $50 \mathrm{~cm}$ mark before the escape interval. Mice that reached the $50 \mathrm{~cm}$ mark during the avoidance interval increased their speed (compared with the pretrial speed) to reach the $50 \mathrm{~cm}$ mark (Fig. 2C; Peak trial vs intertrial speed for Avoids Tukey $\left.t_{(26)}=12.9, p<0.0001\right)$. Mice that failed to reach the $50 \mathrm{~cm}$ mark began moving too late during the avoidance interval. However, all the mice either avoided or escaped successfully on every trial.

Activity of well-isolated neurons was recorded during signaled active avoidance trials; and on the last recording session, a marking lesion was made at the upper edge of the $\mathrm{SNr}$, where cell sampling began (Fig. 2B). The analysis of SNr unit activity focused on avoidance responses, since these are uncontaminated by the effects of the US (presented during the escape interval). $\mathrm{SNr}$ neurons did not robustly activate or deactivate (enhance or decrease firing) during presentation of the ACS before mice began to move to avoid (Fig. 2D, gray). Only a few neurons showed a sharp transient response within the onset of the ACS, but this did not persist with the stimulus. In contrast, movement of the animal during avoidance responses clearly affected $\mathrm{SNr}$ activity. We found three populations of neurons based on their activity during avoidance responses: A group of cells deactivated as the animals avoided (Fig. 2D, left; $n=19$ of 45 neurons; Wilcoxon signed rank $Z=3.8, p<0.0001$ ), a group of cells activated as the animals avoided (Fig. $2 D$, right; $n=10$ of 45 neurons; Wilcoxon signed rank $Z=-2.75, p=0.001$ ), and a group of cells were either not affected or had low firing rates inconsistent with the tonic, high firing rates of $\mathrm{SNr}$ cells ( $n=16$ of 45 neurons). $\mathrm{SNr}$ deactivation and activation closely relate to the movement of the animal, tracking the speed of the animal during the avoidance response (Fig. $2 D$, red). Compared with a pretrial period (2 $s$ before onset of the avoidance interval), both deactivation and activation were significant for a period ( $2 \mathrm{~s}$ before and $1 \mathrm{~s}$ after) around the occurrence of the avoidance response (Fig. 2D). To begin deciphering whether the $\mathrm{SNr}$ deactivation and activation are specifically related to the avoidance movement or to movement in general, we compared SNr firing during the avoidance interval versus the intertrial interval by calculating the cross-correlation between movement speed and $\mathrm{SNr}$ firing during each interval (an equivalent period of $\sim 5 \mathrm{~s}$ per trial was used for each interval pair). In the $\mathrm{SNr}$ cells that deactivated, movement speed was negatively correlated with neuronal firing during both the avoidance interval and the intertrial interval. However, the crosscorrelation was sharper and stronger during the avoidance interval compared with the intertrial interval (Fig. 2E, left; Wilcoxon signed rank $Z=2.87, p=0.002$ ). In the $\mathrm{SNr}$ cells that activated, movement speed was positively correlated with neuronal firing, and this cross-correlation was broader, but similar between both intervals (Fig. 2E, right). The correlations disappeared in shuffled cross-correlograms of the same data (Fig. $2 E$, insets). All crosscorrelations $(-0.5$ to $0.5 \mathrm{~s})$ were significant compared with the corresponding shuffled cross-correlations (Wilcoxon signed rank test, $p<0.0001$ ). Thus, the results indicate that $\mathrm{SNr}$ cells that deactivate during avoidance responses code avoidance speed more robustly and sharply than general (intertrial interval) movement speed, whereas SNr cells that activate code avoidance and general movement speed similarly. This intriguing difference will be explored further in future studies.

The finding that many SNr cells are deactivated during avoidance responses, and that this codes avoidance movement speed robustly, is interesting since we previously showed that inhibiting SNr cells with optogenetics during the avoidance interval drives effective avoidance responses in the absence of an external CS (Hormigo et al., 2016). However, whether the deactivation of SNr cells drives or regulates the neuronal drivers of the avoidance response is unknown. Thus, in the following experiments, we explored the role of $\mathrm{SNr}$ deactivation during signaled active avoidance in a shuttle box.

\section{Inhibition of SNr GABAergic cells serves as an effective CS to drive active avoidance responses}

As already noted, optogenetic inhibition of SNr GABAergic cells during the avoidance interval drives avoidance responses in the absence of an external sensory CS. These results were obtained using Vgat-Arch mice [cross of Vgat-cre and Ai40D mice to express ArchT in GABAergic cells], which broadly express Arch in GABAergic cells throughout the brain (Hormigo et al., 2016). Therefore, we sought to confirm these optogenetic findings with a more selective expression of Arch by bilaterally injecting a Creinducible AAV into the $\mathrm{SNr}$ of Vgat-cre mice [AAV5-EF1aDIO-eArch3.0-EYFP; UNC Vector Core], which limits Arch expression to GABAergic cells in SNr (Vgat-SNr-Arch). Figure $3 A$ shows an example sagittal section of an AAV injection in $\mathrm{SNr}$ from a Vgat-SNr-Arch mouse. The Arch expression was well localized in $\mathrm{SNr}$ with fibers seen coursing toward projection sites of $\mathrm{SNr}$ cells. These animals were implanted with bilateral optical fibers in SNr. Figure 3C shows an example coronal section of a bilateral optical fiber implantation in $\mathrm{SNr}$; note the cannula tracks coursing to SNr. Figure 3D (Movie 1) shows the location of implanted optical fiber endings in $\mathrm{SNr}$ (and PPT for later experiments). The $\mathrm{SNr}$ cannula endings are marked as red circles, and the $\mathrm{SNr}$ volume is traced in semitransparent blue. Also shown for reference are the zona incerta (anterior to $\mathrm{SNr}$ ), the PPT (posterior to SNr), and the superior colliculus (dorsal to $\mathrm{SNr})$.

To verify the effectiveness of the optogenetics approaches, we performed electrophysiological recordings in vivo (anesthetized) and in vitro (acute slices). Whole-cell recordings from $\mathrm{SNr}$ cells in acute slices confirmed that green light was highly effective at suppressing the firing of $\mathrm{SNr}$ cells that express Arch in both Vgat-SNr-Arch and Vgat-Arch mice (Fig. $4 A, B ; n=8$ cells). Several of the whole-cell recorded SNr cells were reconstructed, which shows their typical multipolar dendritic morphology (Fig. $4 A$, right). Continuous pulses of green light (Fig. $4 B$ ) abolished the firing of these cells (Wilcoxon Signed Rank $Z=2.45$, $p=0.007)$. Moreover, in anesthetized mice, unit recordings from SNr cells revealed strong inhibition of $\mathrm{SNr}$ cells surrounding the cannula (Fig. 4C,D). Approximately 55\% (56 of 102) of the sites showed significant inhibition compared with baseline firing (Fig. $4 C$; Wilcoxon Signed Rank $Z=6.5, p<0.0001$, prelight versus during-light per trial comparison), while the other sites were either not affected by the light (36 of 102 sites; 35\%) or their firing was slightly enhanced (10 of 102 sites; 10\%). The excited cells may represent local cells (e.g., substantia nigra pars compacta) that are disinhibited by the synaptic collaterals of suppressed $\mathrm{SNr}$ cells (Tepper et al., 1995; Brown et al., 2014). Some of the 
inhibited cells were tested with longer duration light pulses (5-10 s) to make sure the effect persisted for longer pulse durations, which showed strong inhibition similar to the shorter $1 \mathrm{~s}$ pulses. Figure $4 D$ shows the effect of 5-s-long pulses on the firing of a group of well-inhibited cells ( $n=12$; Wilcoxon Signed Rank $Z=3.02$, $p=0.0004)$. The strong inhibition of firing during the first second of light persisted for the duration of the light pulse; there was no difference between the first second (0-1 s) and the last second (4-5 s) of the light pulse (Wilcoxon Signed Rank $Z=-0.3$, $p=0.7)$.

Since PPT cells are essential for the expression of signaled active avoidance responses, we also recorded from PPT cells in Vgat-SNr-Arch anesthetized mice to determine whether $\mathrm{SNr}$ inhibition disinhibits PPT cells, resulting in PPT excitation during $\mathrm{SNr}$ deactivation. Indeed, we found that many units located in PPT were excited when $\mathrm{SNr}$ cells were inhibited ( $n=58$; Fig. $4 E$, Wilcoxon Signed Rank $Z=-6.6, p<0.0001)$. The inhibited cells were found in a strip that corresponds to the stereotaxic location of PPT. Other cells recorded above, below, and within this strip of PPT were not affected by green light in $\mathrm{SNr}(n=166)$. Thus, both slice and in vivo verifications demonstrate that green light applied in the $\mathrm{SNr}$ of Vgat-SNr-Arch and Vgat-Arch mice robustly inhibits $\mathrm{SNr}$ GABAergic cells (Fig. 4A-D). Importantly, $\mathrm{SNr}$ deactivation disinhibits PPT cells (Fig. 4E).

After confirming that green light inhibits $\mathrm{SNr}$ cells in VgatSNr-Arch mice, which disinhibits cells in PPT, we tested the ability of SNr deactivation to drive avoidance responses by inhibiting $\mathrm{SNr}$ with continuous green light (LCS) during the avoidance interval in substitution of the ACS. Vgat-SNr-Arch mice $(n=5)$ were presented with ACS trials during five daily sessions (Fig. 4F; 1-5 ACS) in which mice readily learned to avoid the US signaled by the ACS. In the next 15 sessions (Fig. $4 F$; 6-20 ACS or LCS), both ACS trials and LCS alone trials were presented randomly (half of each type). The animals also rapidly learned to avoid the US signaled by the LCS. By the time the behavior was firmly established, there were no significant differences between the ACS and LCS alone trials in avoidance rates (Tukey, $p>0.99$ ACS vs LCS alone trials) or other measures (Fig. 4F; 25 sessions in 5 mice; sessions 11 -15 or 1620; latency, intertrial crossings; Tukey, $p>0.98$ ACS vs LCS alone trials). Moreover, when the CS (ACS or LCS) was randomly omitted in some of the trials (No CS trials), performance in those trials dropped sharply to a low avoidance level $(<30 \%$; Tukey $t_{(224)}=31, p<0.0001$ ACS vs No CS trials), indicating that the majority of avoids are in response to the ACS or LCS and not because of random/spontaneous intertrial crossings (Fig. 4F; 13-20 No CS; 40 sessions in 5 mice). Thus, as reported previously for Vgat-Arch mice (Hormigo et al., 2016), we found that $\mathrm{SNr}$ inhibition effectively drives avoidance responses in Vgat-SNr-Arch mice, when Arch expression is limited to GABAergic cells in SNr.
During active avoidance, a black aluminum cap completely covered the head implant where the ferrules connect, blocking any exiting light. To ensure that light alone had no extraneous effects, we implanted Vgat-cre mice that did not express opsins (No Opsin mice: injected with AAV8-hSyn-EGFP, or not injected with an AAV) with bilateral optical fibers in $\operatorname{SNr}(n=4)$. In daily sessions, ACS trials and LCS alone trials were presented randomly. The No Opsin animals learned to avoid the US when it was signaled by the ACS, but not when it was signaled by the LCS. Thus, avoidance rates were much higher during ACS trials than during LCS alone trials (Fig. 4F; No Opsin; 20 sessions in 4 mice; Tukey $\left.t_{(112)}=24, p<0.0001\right)$. Therefore, it is the inhibition of SNr GABAergic cells that drives avoidance responses, not the light causing nonspecific effects in SNr.

\section{Inhibition of SNr GABAergic cells does not drive active avoidance responses in naive mice}

The finding that $\mathrm{SNr}$ inhibition serves as a very effective CS to drive avoidance responses in trained mice raises the question of whether $\mathrm{SNr}$ inhibition innately drives avoidance responses because it causes general movement that leads the animal to shuttle into the other compartment or, alternatively, SNr inhibition requires learning (association with the US) to drive avoidance responses like a true CS. Since optogenetic excitation of the direct pathway can drive movement (Kravitz et al., 2010; Yttri and Dudman, 2016), it is important to dissociate innate motor activity from learned avoidance responses. To address this question, we used No US trials, which are regular ACS or LCS alone trials in which the escape interval is not presented; the avoidance 


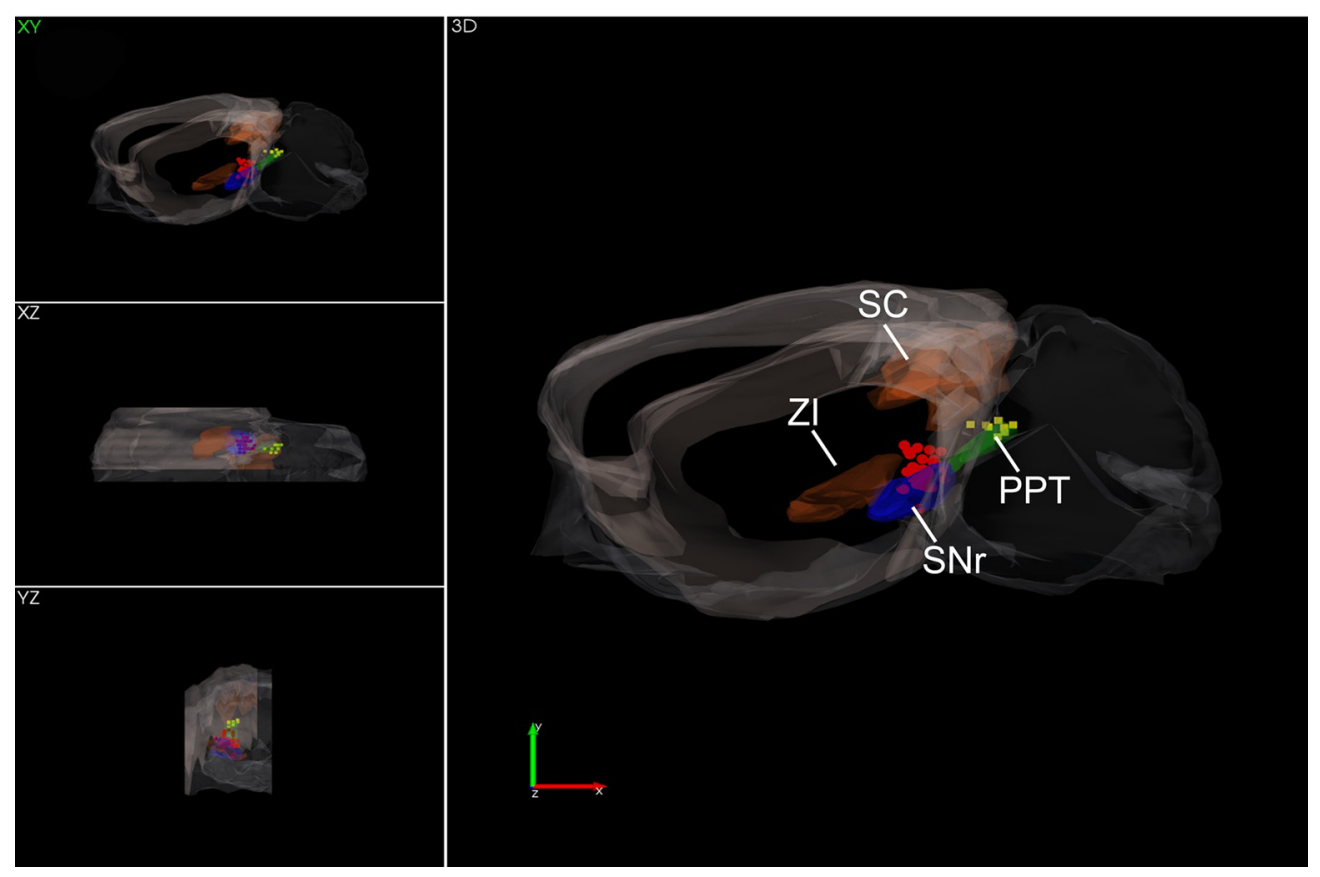

Movie 1. Video of the 3D reconstruction in Figure 3D. [View online]

interval is followed immediately by the intertrial interval when the animal shuttles or when the avoidance interval expires (Fig. $5 A$ ). Thus, we presented No US trials to naive (untrained) animals to test whether mice would innately shuttle to the other compartment when $\mathrm{SNr}$ is inhibited.

Naive (untrained) Vgat-SNr-Arch mice $(n=6)$ were randomly presented with the ACS or the LCS (continuous green light) during No US trials (half of each) for five sessions (Fig. $5 B, C$; No US; 30 sessions in 6 Vgat-SNr-Arch mice). The animals could eliminate the ACS or LCS by shuttling during its presentation. In No US trials, naive animals had low rates of shuttling in response to either the ACS or the LCS (Fig. 5B; Tukey, $p=0.99$ ACS vs LCS in No US trials). These shuttling responses result from normal cage exploration and movement; thus, SNr inhibition does not cause naive mice to shuttle.

During the next 10 sessions (Fig. 5B; Include US), regular ACS and LCS alone trials were randomly presented (half of each) using the AA1 procedure. The animals readily learned to avoid the US in response to the ACS or the LCS. The rate of learning was not significantly different between ACS trials or LCS alone trials. There was no difference in avoidance rates (Tukey, $p>$ 0.97 ACS vs LCS alone trials), latency or intertrial crossings (Fig. $5 B$; Tukey, $p>0.33$ ACS vs LCS alone trials) between ACS and LCS alone trials. In No CS trials presented during some of the sessions (Fig. 5B; No CS), avoidance rates dropped sharply, indicating that the animals rely on the ACS and the LCS to drive avoidance responses. Video tracking showed that trial speed and velocity increased sharply during learning for both ACS or LCS alone trials while intertrial speed remained the same (Fig. $5 C$ ), indicating that the mice move quickly in response to either CS.

In conclusion, $\mathrm{SNr}$ inhibition does not drive shuttling in naive mice. Like a true CS, SNr inhibition only begins to drive avoidance responses when SNr inhibition predicts the US.

Inhibition of $\mathrm{SNr}$ GABAergic cells serves as an effective CS to drive passive avoidance responses

The previous results indicate that inhibition of SNr GABAergic cells can serve as an effective CS signal to drive active avoidance responses. Next, we tested whether $\mathrm{SNr}$ inhibition could be used as an effective CS signal to drive the opposite response, a passive avoidance response, by which mice must inhibit their shuttling response during the avoidance interval. For this, we used the AA3 procedure where animals must actively avoid in response to one CS (ACS) and passively avoid in response to another CS (LCS; Fig. 5A).

Initially, the mice were trained to actively avoid in response to both the ACS and LCS (Fig. 5B,C; Include US) and afterward subjected to AA3 (Fig. 5B,C; AA3). In AA3, the contingency of the LCS trials changed, so the animals must not shuttle during the avoidance interval signaled by the LCS to passively avoid the US. In other words, in the AA3 procedure, mice are required to change their response to the LCS from an active avoidance response to a passive avoidance response. Mice rapidly learned to discriminate between the ACS and the LCS and inhibited their shuttling responses during presentation of the LCS while continuing to actively avoid in response to the ACS (Tukey $t_{(45)}=$ $60, p<0.00001$ ACS vs LCS; active avoids in AA3). There was a reduction in trial speed during AA3 compared with regular ACS and LCS active avoidance trials, and this reduction was stronger for LCS trials since the mice learned not to move in response to the LCS (Include US vs AA3; Fig. 5C; Tukey $t_{(45)}=22$, $p<0.0001$ ACS vs LCS in AA3). No Opsin mice did not learn to actively avoid the LCS (Fig. $4 F$ ); thus, they were not tested in the AA3 procedure that would require them to passively avoid the LCS. These results indicate that $\mathrm{SNr}$ deactivation can function as an effective CS to drive either an active or a passive avoidance response.

Direct striatonigral pathway activation in $\mathrm{SNr}$ serves as an effective CS to drive active avoidance responses...

The direct striatonigral pathway is the main source of GABAergic input to SNr cells (Albin et al., 1989; DeLong, 1990; Smith et al., 1998; Hikida et al., 2010; Kravitz et al., 2010; Kravitz and Kreitzer, 2012; Tai et al., 2012; Cui et al., 2013; Freeze et al., 2013; Tecuapetla et al., 2014). Since direct inhibition of SNr cells with Arch drives avoidance responses, we tested whether exciting 
A

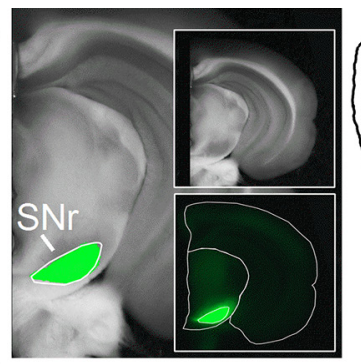

C

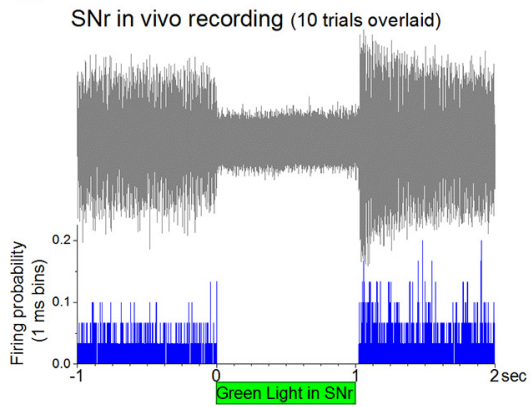

E
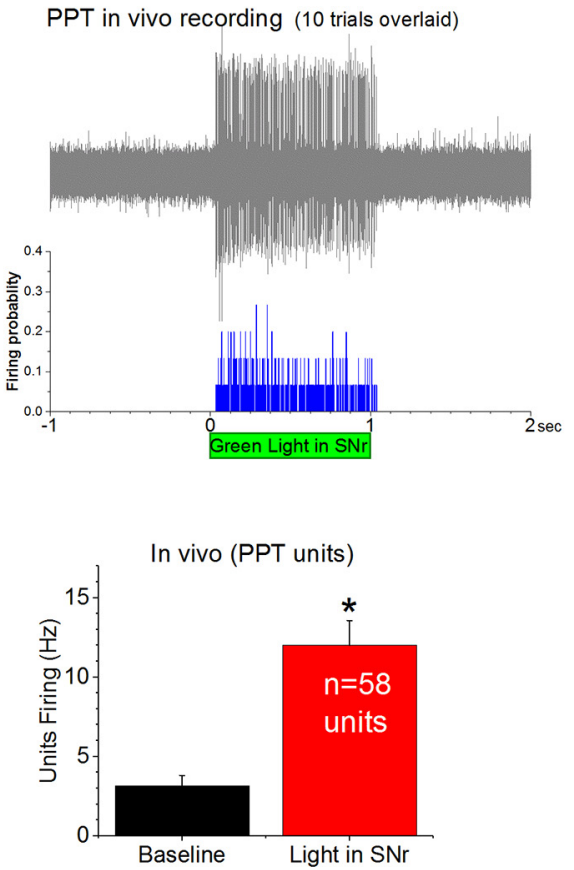

B
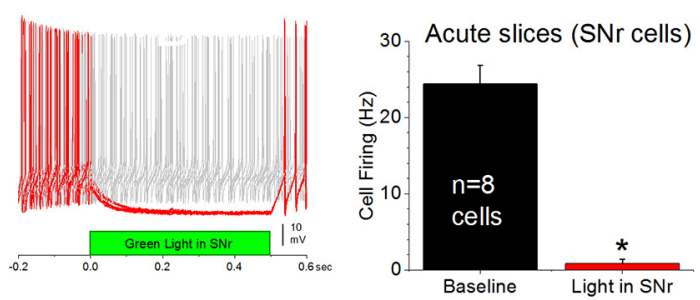

D
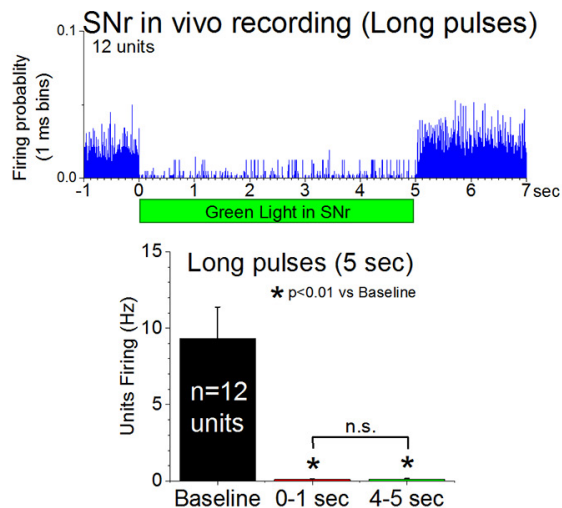

$\mathbf{F}$

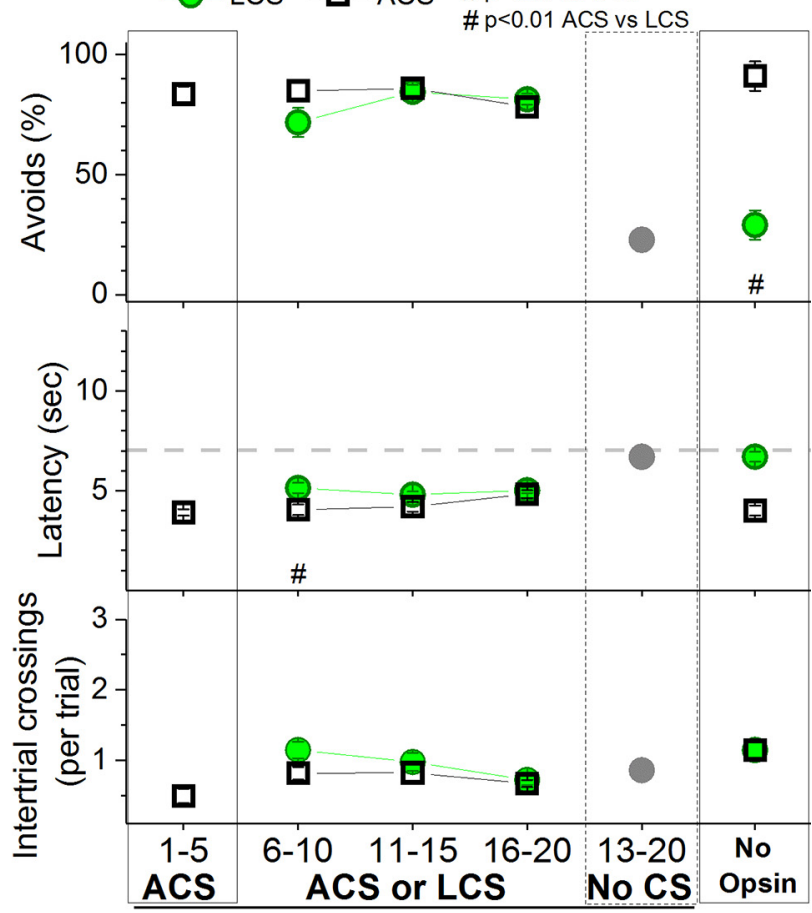

Blocks of Sessions

Figure 4. Optogenetic inhibition of SNr GABAergic cells. A, Left, Coronal section showing YFP fluorescence in a Vgat-Arch slice expressing ArchT completely filling the SNr. The main image blends a bright-field image of the slice with the green channel of the eYFP fluorescence image. Insets, The bright-field (top) and the fluorescence image (bottom) alone. Right, Reconstruction of SNr cells that were whole-cell recorded in slices to test the effects of green light in Vgat-Arch and Vgat-SNr-Arch mice. B, Left, Example showing the effect of green light on the firing of a whole-cell recorded SNr cell from a Vgat-SNr-Arch mouse slice (in vitro). The overlaid traces (6 per condition) show spontaneous firing (gray) and the effect of a continuous pulse of green light (red). Note the robust inhibition of cell firing. Right, Population data obtained from a group of SNr cells inhibited by green light in Vgat-SNr-Arch mice. C, Left, Example of the effect of green light (1 s pulses) on SNr unit firing tested in an isoflurane-anesthetized Vgat-SNr-Arch mouse in vivo. Plot represents overlaid raw traces (10 trials) and a PSTH (30 trials). Note the robust inhibition of $\mathrm{SNr}$ cell firing during the continuous green light pulse. Right, Population data obtained from a group of SNr units $(n=56$ of 102 recording sites surrounding 6 implanted optical fibers in 3 mice) inhibited by continuous green light (25-35 mW) in Vgat-SNr-Arch mice. D, Effect of longer duration green light pulses on SNr unit firing inhibition in isoflurane-anesthetized VgatSNr-Arch mice in vivo. Top, Population PSTH of SNr units $(n=12)$ inhibited by a $5 \mathrm{~s}$ continuous pulse of green light. Bottom, Comparison between baseline firing and the first or last second of a $5 \mathrm{~s}$ green light pulse. The inhibition persists during the long duration pulses; the amount of inhibition does not differ between the first and last second of the $5 \mathrm{~s}$ pulse. $\boldsymbol{E}$, Top, Example of the effect of green light applied in the SNr of an isoflurane-anesthetized Vgat-SNr-Arch mouse in vivo on the firing of a cell in PPT. Bottom, Population data obtained from PPT units ( $n=58$ ) excited when SNr GABAergic cells are inhibited with green light applied in the SNr of Vgat-SNr-Arch mice. $\boldsymbol{F}$, Vgat-SNr-Arch mice $(n=5)$ that trained to avoid the US signaled by the ACS learn rapidly to avoid the US signaled by SNr inhibition caused by the LCS (continuous green light in SNr). Plots represent the percentage of avoidance responses (top), response latency from trial onset (middle), and the number of intertrial crossings per trial (bottom) for blocks of five sessions (AA1). During the first block of sessions (1-5), animals were trained with ACS trials to avoid the US signaled by the ACS. During the next three blocks of sessions (6-20), the animals received ACS and LCS alone trials (half of each). In these sessions, there were no significant differences 


\section{A AA3 procedure}
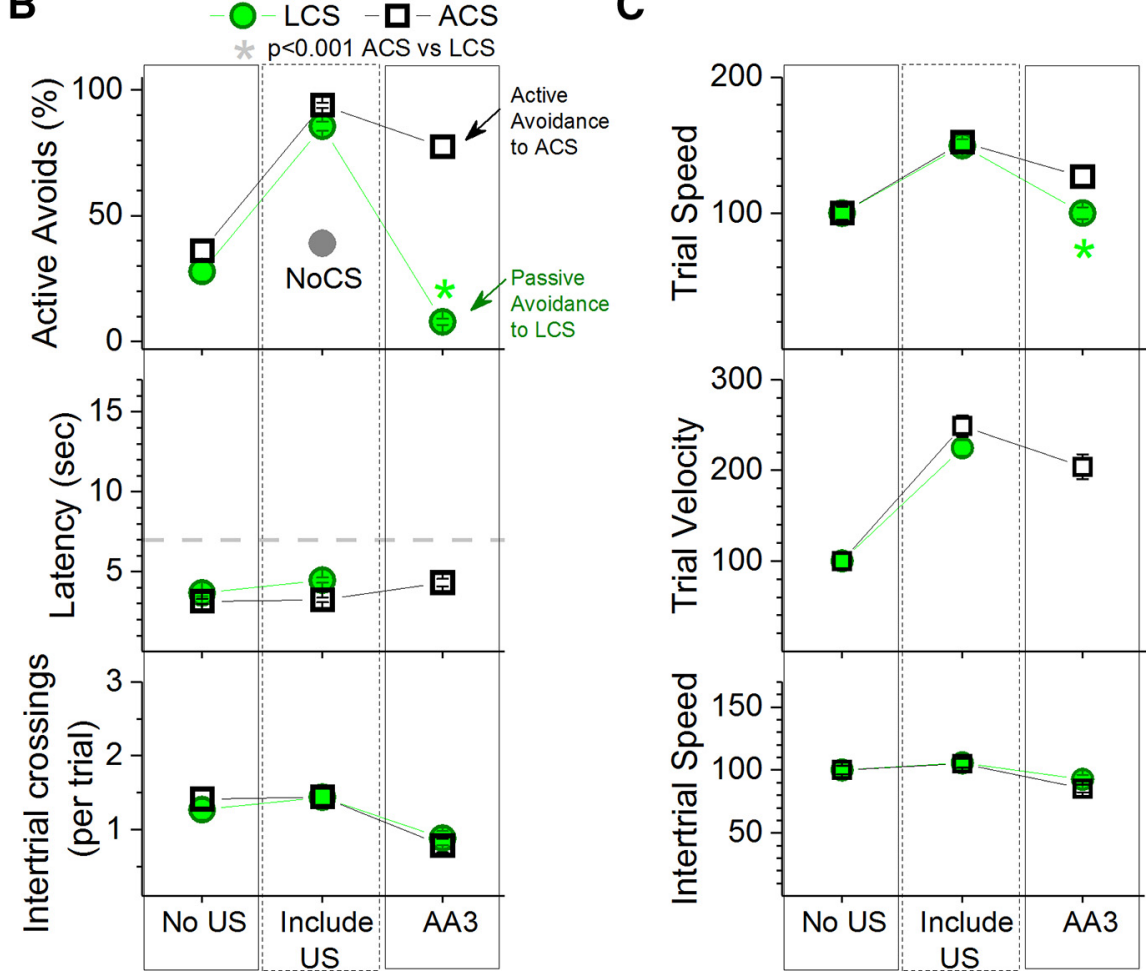

Figure 5. SNr inhibition is an effective $C S$ to drive active and passive avoidance responses. $A$, Schematic of the AA3 procedure used to test the ability of SNr inhibition to drive passive avoidance responses. In AA3, ACS and LCS trials are presented randomly, but LCS trials require mice to passively avoid by not shuttling during the presentation of the LCS. If animals shuttle during the LCS-signaled passive avoidance interval, they are punished ( $0.5 \mathrm{~s}$ foot-shock and white noise [WN]). $\boldsymbol{B}$, Plots (details in Fig. 4F) show three blocks of sessions for the same mice. In the first block (No US), naive (untrained) mice $(n=6)$ were presented with ACS and LCS alone trials that did not include a US (escape interval). The naive animals did not learn to shuttle during presentation of the ACS or the LCS in the absence of a US and the shuttling did not differ between these trials. During the next block of sessions (Include US), the animals received ACS and LCS alone trials that included a US (AA1); No CS trials were also presented. The animals learned to avoid the US when the ACS and LCS began to predict the US. There was no difference in avoidance rates or other measures between ACS and LCS alone trials. During the last block of sessions (AA3), the AA3 procedure was applied, which requires passive avoidance in LCS trials (instead of the active avoidance required in the previous sessions). Mice rapidly learned passive avoidance signaled by the LCS, while continuing active avoidance signaled by the ACS. C, Trial speed, trial velocity, and intertrial speed for the data in $\boldsymbol{B}$. Trial velocity is not available for LCS trials in AA3 since there is no effective direction in passive avoidance. In AA3, trial speed was lower in LCS trials compared with ACS trials, since the mice learned not to move in response to the LCS.

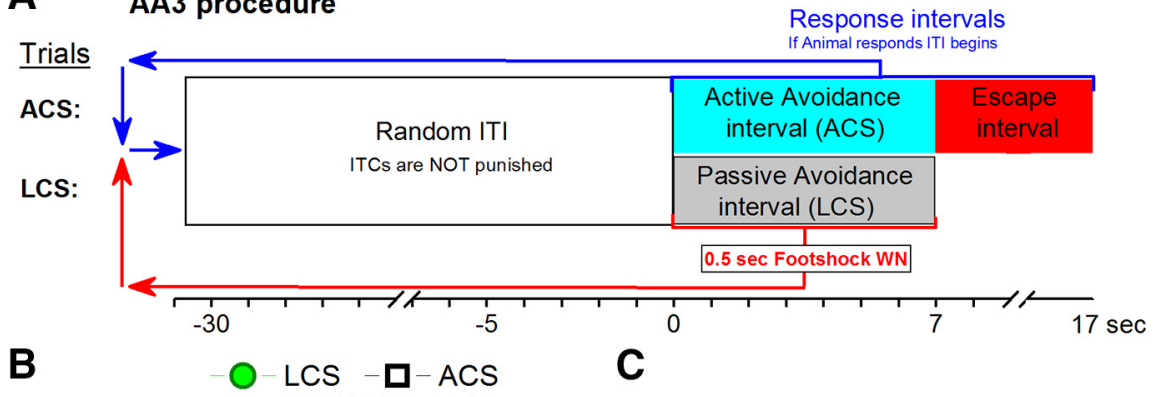

GABAergic striatonigral fibers in $\mathrm{SNr}$ would also drive avoidance responses. Bilateral injections of a Cre-inducible AAV [AAV5-EF1a-DIO-hChR2(H134R)eYFP; UPenn vector core] were performed in the striatum of Vgat-Cre mice to express channelrhodopsin-2 (ChR2) in GABAergic cells located in different parts of the striatum. In response to blue light applied in $\mathrm{SNr}$, ChR2 excites the fibers of the GABAergic striatum cells that project to $\mathrm{SNr}$ and, consequently, synaptically inhibits the contacted $\mathrm{SNr}$ cells. Three portions of the striatum were targeted with single AAV injections: the ventral striatum (Vgat-StrV-ChR2), the dorsal striatum (Vgat-StrD-ChR2), and the NAc (Vgat-Acb-ChR2), which is ventromedial. An additional group of animals received dual injections targeting both the dorsal and ventral striatum (Vgat-StrDV-ChR2). The injections in striatum resulted in the expression of $\mathrm{ChR} 2$ in striatonigral fibers innervating $\mathrm{SNr}$ in the midbrain (Fig. 6A$D)$. These mice were implanted with bilateral optical fibers in $\mathrm{SNr}$ (Fig. 3C,D) to stimulate GABAergic striatonigral synapses originating in the ventral or dorsal striatum (Fig. 6E). The optogenetic stimulation was blue light $(0.4-1.1 \mathrm{~mW})$ delivered as continuous pulses or trains of $1 \mathrm{~ms}$ pulses at different frequencies.

We used LCS alone trials to determine whether inhibiting $\mathrm{SNr}$ neurons synaptically, by exciting striatonigral GABAergic fibers, is an effective CS to drive active avoidance responses, just like directly inhibiting $\mathrm{SNr}$ neurons with Arch. In the plots for LCS alone sessions (Fig. 7A,B), we highlight with nonsignificant differences (\#) between LCS alone trials and ACS trials to denote LCS that are equivalent to the ACS at driving avoidance responses. We found that excitation of striatal fibers in $\mathrm{SNr}$ that originate in either the ventral striatum (Fig. $7 A$, open black squares; 64 sessions in 4 mice) or the dorsal striatum (Fig. $7 \mathrm{~A}$, open green circles; 55 sessions in 4 mice) was very effective at driving avoidance responses. These animals had average avoidance rates $>60 \%$ for most LCS alone trials (including low-frequency blue light trains at 2 and $5 \mathrm{~Hz}$ ), while No

between the ACS and LCS alone trials in avoidance rates or other measures. During the last 2 blocks, No CS trials were also presented (13-20). In No CS trials, performance was significantly impaired compared with ACS trials. The No Opsin column represents a group of control animals that do not express Arch (No Opsin, $n=4$ ) but were subjected to the same LCS and ACS trials during an equivalent number of sessions. The LCS was ineffective at driving avoidance responses in these mice; responding levels were similar to No CS trials. Thus, in these mice, avoidance rates were much higher during ACS trials than during LCS alone trials. n.S., not significant.
CS trials did not reach $30 \%$ avoidance. Stimulation of striatonigral synapses originating in the ventral striatum was particularly effective at driving avoidance responses in LCS alone trials that used blue trains at 10,20 , and $100 \mathrm{~Hz}$; avoidance rates were equivalent to ACS trials (Fig. 7A, top). Response latency for LCS alone trials was generally slightly slower than for ACS trials, suggesting that they process the LCS differently than the ACS (Fig. $7 A$, middle). Intertrial crossings were not affected by the LCS alone trials (Fig. $7 A$, bottom). The main repeated-measures 

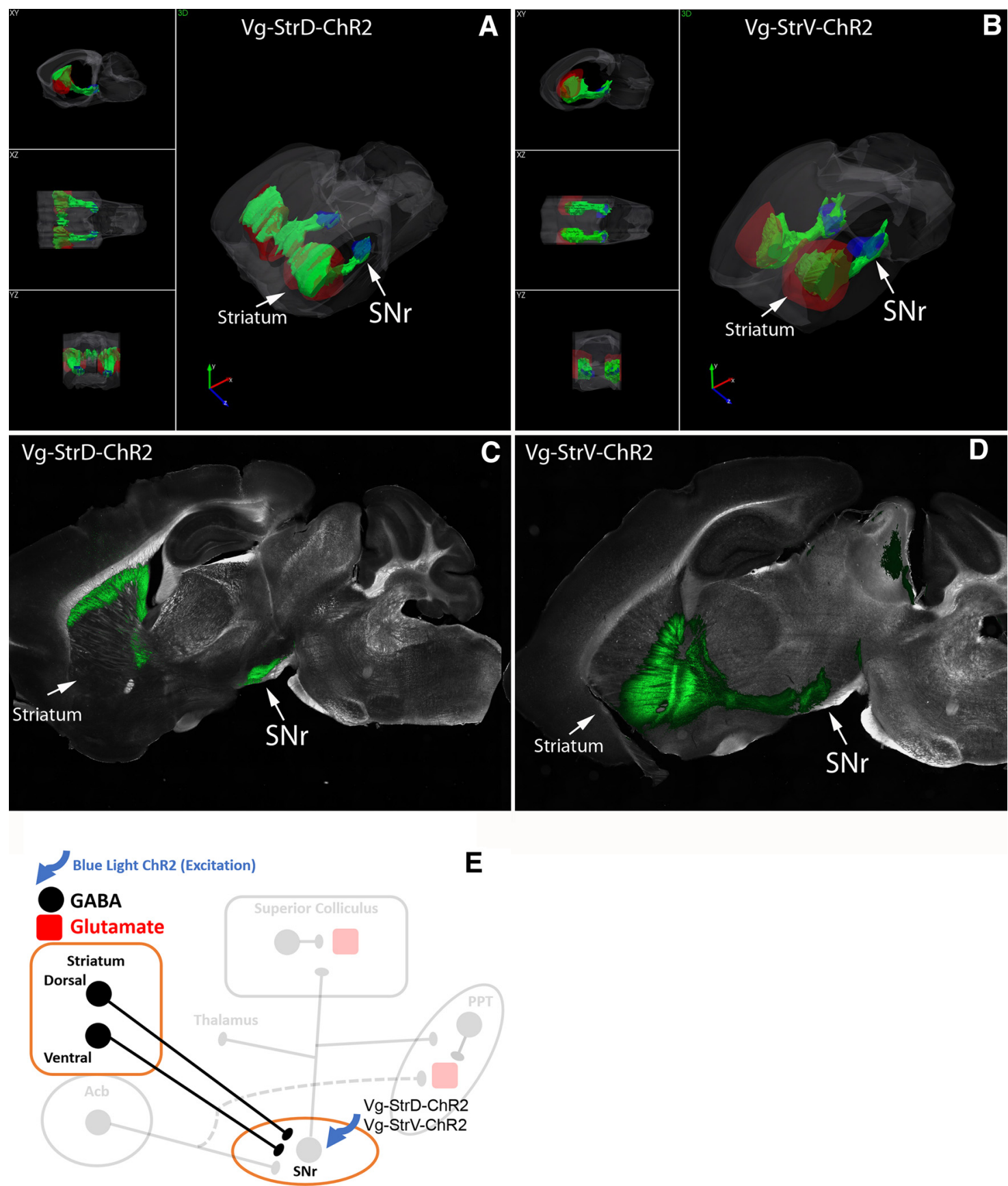

Figure 6. 3D reconstructions showing typical results of AAV injections to express ChR2 in GABAergic cells of the dorsal striatum $(\boldsymbol{A})$ and ventral striatum $(\boldsymbol{B})$. In these experiments, cannulas were implanted in SNr. All sites showing some level of eYFP expression are labeled (green), which reflects both the cells labeled at the injection site and all the projecting fibers. The striatum and $\mathrm{SNr}$ are filled in semitransparent red and blue, respectively. The images are placeholders for Movies 2 and 3, respectively. C, D, Example parasagittal sections from two different mice showing eYFP fluorescence after injection in dorsal $(\boldsymbol{C})$ or ventral $(\boldsymbol{D})$ striatum. The images blend a dark-field image of the section with the green channel of the eYFP fluorescent image. Note the fluorescence in striatum (injection site) and in SNr (projection site). $\boldsymbol{E}$, The schematic highlights the pathways targeted by optogenetics in the experiments shown in Figure 7. Two different GABAergic striatonigral pathways (black) originating in the dorsal or ventral striatum were targeted. During active avoidance, striatonigral fibers were excited by activating ChR2 with blue light applied in the SNr through optical fibers (blue arrow). Semitransparent structures refer to other pathways/areas manipulated in this or in our previous work.

factor of the mixed ANOVA [ $F$ and $p$ values] and the Tukey test $p$ values for LCS alone trials that are equivalent (i.e., not significantly different) compared with ACS trials are indicated here for each measure per group. Vgat-StrV-ChR2: Avoids $\left[F_{(8,480)}=\right.$ 23.8, $p<0.0001], p=0.7(10 \mathrm{~Hz}), p=0.73(20 \mathrm{~Hz}), p=0.34$ $(100 \mathrm{~Hz})$. Latency $\left[F_{(8,480)}=36.9, p<0.0001\right]$, none. Intertrial crossings $\left[F_{(8,480)}=5, p<0.0001\right], p>0.2(10-100 \mathrm{~Hz}$ and continuous). Vgat-StrD-ChR2: Avoids $\left[F_{(8,408)}=22.7, p<0.0001\right]$, none. Latency $\left[F_{(8,408)}=15, p<0.0001\right]$, none. Intertrial crossings $\left[F_{(8,408)}=3.4, p<0.0007\right], p>0.4(2-100 \mathrm{~Hz}$ and continuous). Vgat-StrDV-ChR2: Avoids $\left[F_{(8,232)}=35, p<0.0001\right]$, $p=0.79(10 \mathrm{~Hz}), p=0.06(20 \mathrm{~Hz}), p=0.02(100 \mathrm{~Hz})$. Latency $\left[F_{(8,232)}=27.2, p<0.0001\right], p=0.3(10 \mathrm{~Hz})$. Intertrial crossings $\left[F_{(8,232)}=7.1, p<0.0001\right], p>0.02(2-100 \mathrm{~Hz}$ and continuous $)$.

However, for both of these groups of mice, there was a small, but noticeable, impairment in driving avoidance responses when the LCS alone trials used blue light trains at $\sim 40 \mathrm{~Hz}$. This impairment at $40 \mathrm{~Hz}$ was more notorious when we excited 


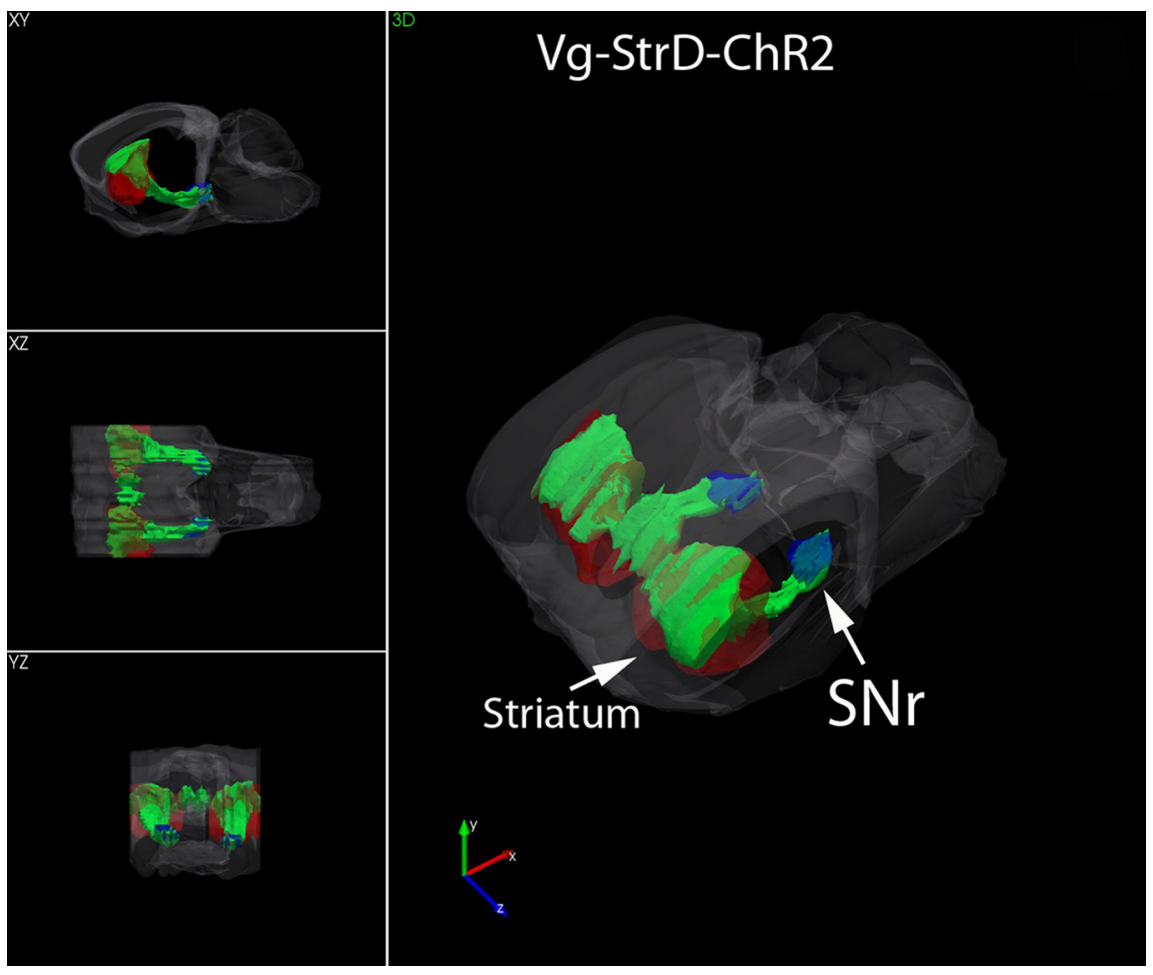

Movie 2. Video of the 3D reconstruction in Figure 6A. [View online]

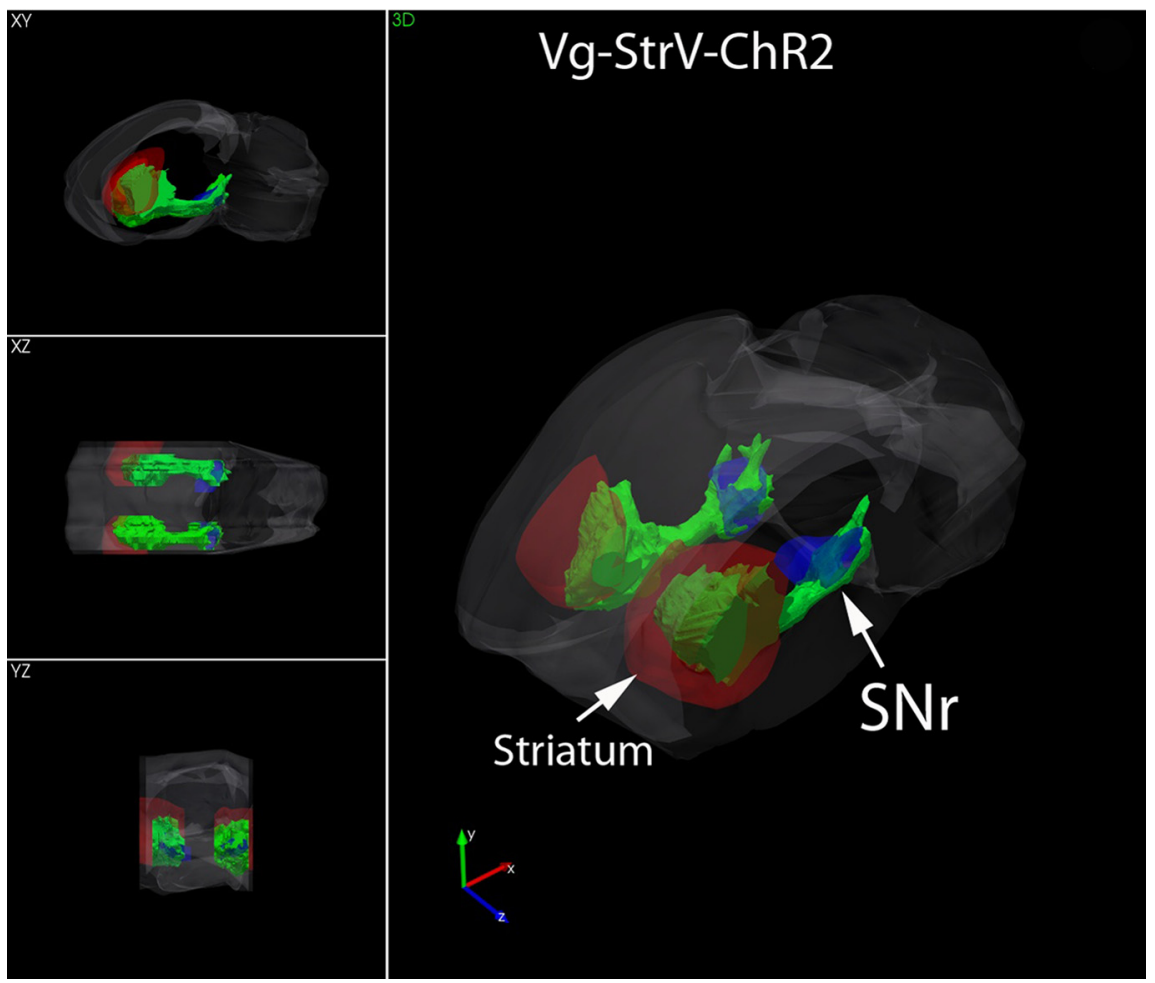

Movie 3. Video of the $3 D$ reconstruction in Figure $6 B$. [View online]

striatal fibers in $\mathrm{SNr}$ originating in a broader region of striatum, encompassing both dorsal and ventral striatum (Fig. $7 A$, closed blue circles; 33 sessions in 4 mice), perhaps because of synaptic cooperativity caused by stimulating more fibers. Thus, excitation of striatal fibers in $\mathrm{SNr}$ at $\sim 40 \mathrm{~Hz}$ appears to cause neuronal activity that impairs active avoidance. Moreover, video tracking of these three groups during the active avoidance sessions (Fig. $7 B$ ) revealed that trial velocity was significantly suppressed in all three groups of animals when the LCS alone trials consisted of blue light trains at $40-66 \mathrm{~Hz}$ (Fig. 7B, middle), but trial speed was not suppressed (Fig. $7 B$, top). The fact that trial speed was not suppressed but trial velocity was suppressed indicates that, during LCS alone trials at 40$66 \mathrm{~Hz}$, animals move as much as during ACS trials (control trials), but they do not move in the correct direction to avoid the US (toward the safe compartment of the cage). Intertrial speed was generally not affected by the LCS alone trials in any of the groups, indicating that the effects of the light occurred selectively when the light was present during each trial and not between trials (Fig. $7 B$, bottom). In No Opsin mice (Fig. 7A, open orange triangles; 28 sessions in 4 mice), LCS alone trials were ineffective at driving avoidance responses compared with ACS and NoCS trials, and the effect of the different light patterns was averaged since they did not differ (Fig. $7 A$ ). This indicates that it is the activation of ChR2, not extraneous effects of the light, that drives the avoidance responses. The main repeated-measures factor of the mixed ANOVA [ $F$ and $p$ values] and the Tukey test $p$ values for LCS alone trials that are equivalent (i.e., not significantly different) compared with ACS trials are indicated here for each measure per group. Vgat-StrV-ChR2: Trial speed $\left[F_{(8,480)}=12\right.$, $p<0.0001], p>0.06(5,20,66,100 \mathrm{~Hz}$ and continuous). Trial velocity $\left[F_{(8,480)}=\right.$ 23.3, $p<0.0001$ ], $p>0.05$ (5 Hz and continuous). Intertrial speed $\left[F_{(8,480)}=4.9\right.$, $p<0.0001], p>0.56(2-100 \mathrm{~Hz}$ and continuous). Vgat-StrD-ChR2: Trial speed $\left[F_{(8,392)}=7.7, p<0.0001\right], p>0.2(10,40$ $100 \mathrm{~Hz}$ and continuous). Trial velocity $\left[F_{(8,392)}=5.1, p<0.0001\right], p>0.11(10$, $20,100 \mathrm{~Hz}$ and continuous). Intertrial speed $\left[F_{(8,392)}=7.5, p<0.0001\right], p>0.02$ $(2-100 \mathrm{~Hz}$ and continuous). Vgat-StrDVChR2: Trial speed $\left[F_{(8,232)}=6, p<\right.$ 0.0001 ], $p>0.3$ (2-100 HZ and continuous). Trial velocity $\left[F_{(8,232)}=10.6, p<\right.$ $0.0001], p>0.08(2-20,100 \mathrm{~Hz})$. Intertrial speed $\left[F_{(8,232)}=0.8, p=0.5\right], p>0.7(2-$ $100 \mathrm{~Hz}$ and continuous).

...but exciting direct pathway fibers in $\mathrm{SNr}$ selectively at $\sim 40 \mathrm{~Hz}$ impairs signaled active avoidance

The previous results (Fig. $7 A, B$ ) show that exciting direct pathway striatal GABAergic fibers in $\mathrm{SNr}$ serves as an effective $\mathrm{CS}$ to 
drive avoidance responses. This agrees with the finding that directly inhibiting $\mathrm{SNr}$ cells with Arch also serves as an effective CS to drive avoidance responses (Fig. 4F). Interestingly, most patterns of blue light stimulation used to excite striatonigral fibers in $\mathrm{SNr}$ were an effective CS to drive avoidance responses, with the exception of stimulation at $\sim 40 \mathrm{~Hz}$, particularly when fibers originating in a broader region of striatum were excited. There seems to be an active suppression of avoidance responses when fibers are excited at $\sim 40 \mathrm{~Hz}$. To test this possibility, we conducted additional sessions in which ACS trials were compared with ACS + LCS trials (Fig. 7C). In ACS + LCS trials, the avoidance interval is signaled by the same ACS as in ACS trials while optogenetic light is simultaneously delivered into the brain bilaterally, and the light persists during the escape interval. The purpose of ACS + LCS sessions is to determine whether the optogenetic manipulation has any effect on avoidance responses driven by the ACS. Thus, in ACS + LCS sessions (Fig. 7C,D; see Figs. $9 E, 12 A, B)$, we indicate significant differences $(* p<0.01)$ between ACS + LCS trials and ACS trials to highlight optogenetics stimuli that affect responses driven by the ACS.

In ACS + LCS trials, stimulation of striatal fibers in $\mathrm{SNr}$ originating in the ventral (Fig. 7C, green open circles; 26 sessions in 4 mice) or dorsal (Fig. $7 C$, black open squares; 27 sessions in 4 mice) striatum produced only a modest suppression of avoidance responses driven by the ACS. With both ventral and dorsal stimulation, avoidance rates were suppressed (Fig. 7C, top) and response latencies were increased (Fig. $7 C$, middle) by blue light trains at $40-66 \mathrm{~Hz}$, while intertrial crossings were not affected (Fig. 7C, bottom). However, excitation of striatal fibers in $\mathrm{SNr}$ originating in a broader region of striatum, encompassing both the dorsal and ventral striatum (Fig. $7 C$, closed blue circles; 28 sessions in 4 mice), produced a much stronger suppression of avoidance responses and increased response latencies when blue light trains were delivered at $20-100 \mathrm{~Hz}$. Intertrial crossings were mostly unaffected for this group, but increased slightly only for $40 \mathrm{~Hz}$ trials, which also produced the strongest impairment in avoidance. This reflects the fact that mice tend to increase their intertrial crossings after being shocked in the preceding escape interval because of failure to avoid in the trial. The main repeated-measures factor of the mixed ANOVA $[F$ and $p$ values] and the Tukey test $p$ values for
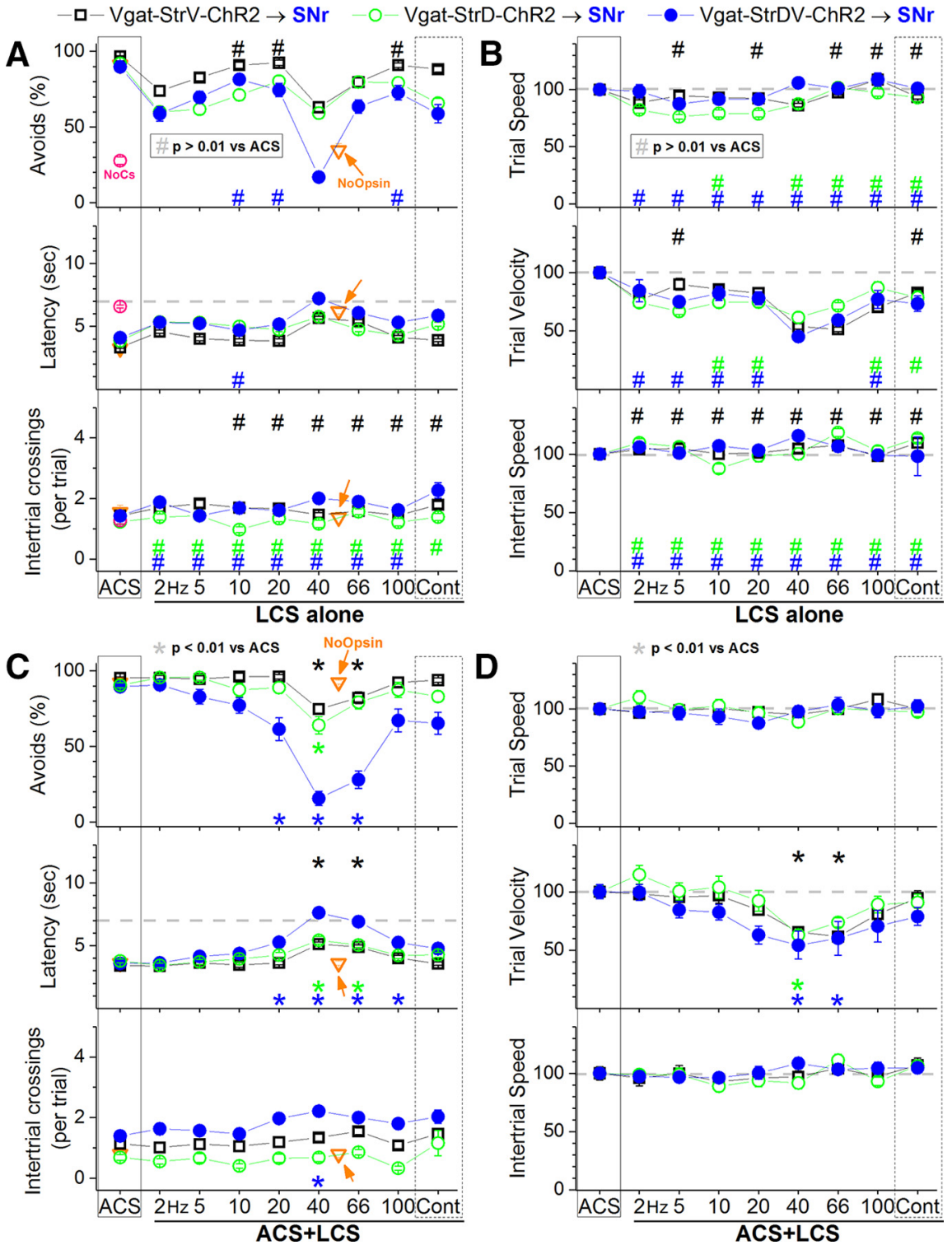

Figure 7. Effect of striatonigral pathway activation in SNr on signaled active avoidance responses. $\boldsymbol{A}$, LCS alone trial sessions to see effects of blue light applied in the SNr on animals that express ChR2 in striatal GABAergic fibers originating in ventral (Vgat-StrV-ChR2), dorsal (Vgat-StrD-ChR2), or both (Vgat-StrDV-ChR2) portions of the striatum (33-55 sessions in 4 mice/ group). The percentage of avoidance responses (top), response latency from trial onset (middle), and the number of intertrial crossings per trial (bottom) are shown. No CS trials (red) were also presented and are averaged for all sessions and shown in the first, ACS column. In LCS alone sessions: "Nonsignificant differences versus ACS trials to denote equivalency in the ability of the optogenetic light to drive avoidance responses. Also shown are the results from a group of No 0psin mice subjected to the same procedures (open triangles combine the different optogenetic stimulation patterns since they did not differ). $\boldsymbol{B}$, Trial speed, trial velocity, and intertrial speed for the data in $\boldsymbol{A}$. $\boldsymbol{C}$, Effect of blue light applied in the SNr on ACS +LCS trial sessions for the groups in $\boldsymbol{A}$ (26-29 sessions in 4 mice). In ACS + LCS sessions: * Significant differences versus ACS trials to denote the effect of the optogenetic light on the ability of the ACS to drive avoidance responses. Also shown are the results from a group of No Opsin mice subjected to the same procedures (open triangles combine the different optogenetic stimulation patterns since they did not differ). D, Trial speed, trial velocity, and intertrial speed for the data in $\boldsymbol{C}$.

ACS + LCS trials that are significantly different compared with ACS trials are indicated here for each measure per group. VgatStrV-ChR2: Avoids $\left[F_{(8,176)}=11.7, p<0.0001\right], p<0.00001$ $(40-66 \mathrm{~Hz})$. Latency $\left[F_{(8,176)}=21.4, p<0.0001\right], p<0.00001(40-66$ $\mathrm{Hz})$. Intertrial crossings $\left[F_{(8,176)}=4.9, p<0.0001\right]$, none. Vgat-StrDChR2: Avoids $\left[F_{(8,184)}=11.4, p<0.0001\right], p<0.00001(40 \mathrm{~Hz})$. Latency $\left[F_{(8,184)}=16.5, p<0.0001\right], p<0.00001 \quad(40-66 \mathrm{~Hz})$. Intertrial crossings $\left[F_{(8,184)}=2.6, p=0.01\right]$, none. Vgat-StrDV-ChR2: Avoids $\left[F_{(8,192)}=54, p<0.0001\right], p<0.0009(20-66 \mathrm{~Hz})$. Latency 

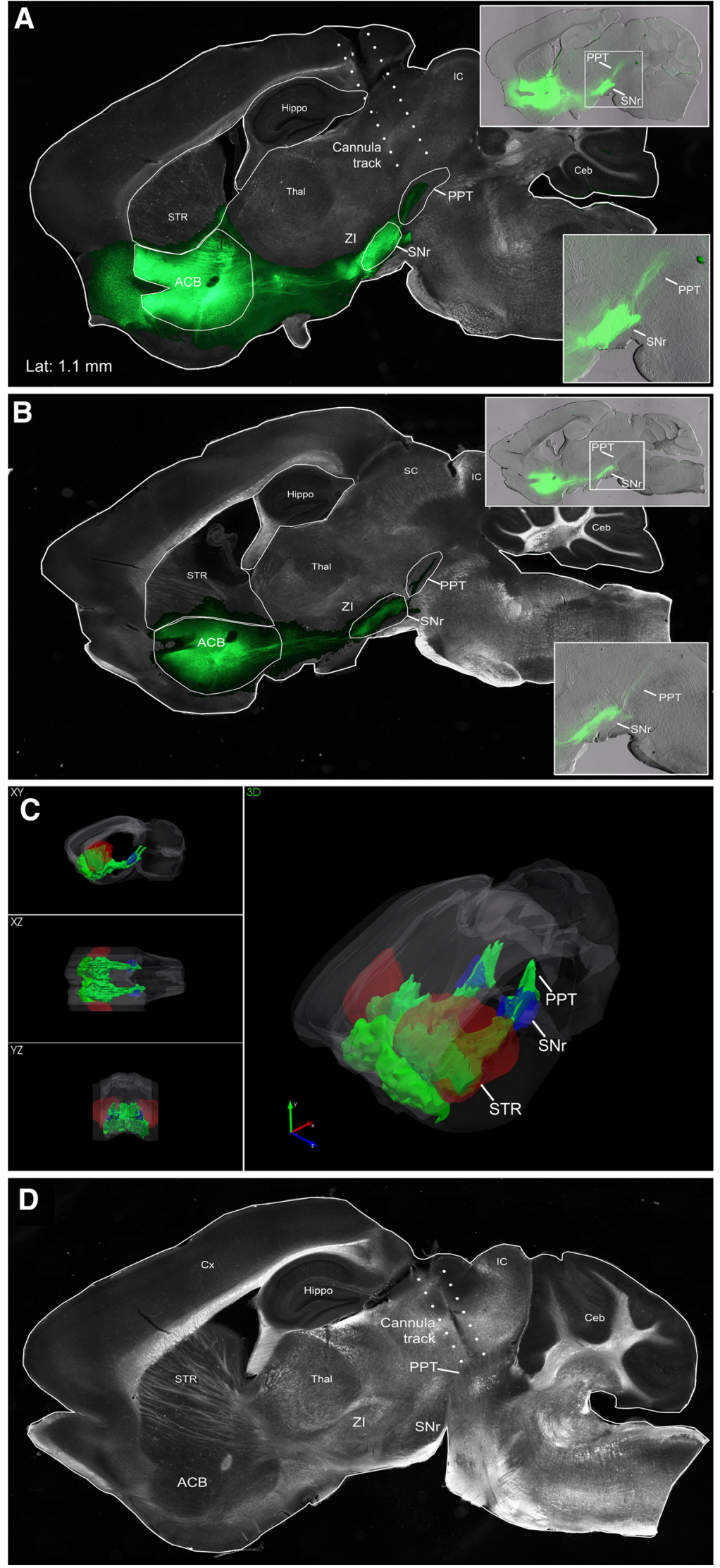

Figure 8. Location of AAV injections in NAc to express ChR2 in GABAergic neurons. $A, B$, Example parasagittal sections from 2 animals with typical ChR2 expression in NAc and corresponding projections to SNr and PPT after an AAV injection in Vgat-Acb-ChR2 mice. Top right insets, An image of the maximal intensity projection of the fluorescence. Bottom right insets, Closeup of the top inset around SNr and PPT. Note the eYFP fluorescent fibers
$\left[F_{(8,192)}=56.4, p<0.0001\right], p<0.00001(20-100 \mathrm{~Hz})$. Intertrial crossings $\left[F_{(8,192)}=4.5, p<0.0001\right], p<$ $0.0001(40 \mathrm{~Hz})$.

Video tracking revealed that trial and intertrial speed was not affected by exciting striatal fibers in the $\mathrm{SNr}$ of any of the three groups (Fig. $7 D$, top, bottom). However, the impairment in avoidance responses with blue light trains at $40-66 \mathrm{~Hz}$ was associated with a significant suppression of trial velocity in ACS + LCS trials for the three groups (Fig. $7 D$, middle). Thus, when avoidance responses are impaired by exciting striatal fibers in $\mathrm{SNr}$ with blue light trains at $40-66 \mathrm{~Hz}$, animals move as much as during control ACS trials but not in the correct direction to avoid. In No Opsin mice (Fig. 7C, open orange triangles; 20 sessions in 4 mice), LCS + ACS trials had no effect compared with ACS trials, indicating that it is the activation of ChR2 at $\sim 40 \mathrm{~Hz}$, not extraneous effects of the light, that impairs avoidance responses driven by the ACS. Therefore, the results show that exciting striatal fibers in $\mathrm{SNr}$ selectively at $\sim 40 \mathrm{~Hz}$ impairs signaled active avoidance driven by the ACS (Fig. 7C,D). The main repeated-measures factor of the mixed ANOVA $[F$ and $p$ values] and the Tukey test $p$ values for ACS + LCS trials that are different compared with ACS trials are indicated here for each measure per group. Vgat-StrV-ChR2: Trial speed $\left[F_{(8,176)}=2.8\right.$, $p=0.01]$, none. Trial velocity $\left[F_{(8,176)}=14.7\right.$, $p<0.0001], p<0.00001(40-66 \mathrm{~Hz})$. Intertrial speed $\left[F_{(8,176)}=3.7, p=0.0004\right]$, none. Vgat-StrD-ChR2: Trial speed $\left[F_{(8,168)}=3, p=0.002\right]$, none. Trial velocity $\left[F_{(8,168)}=11.2, p<0.0001\right], p<0.0001(40 \mathrm{~Hz})$. Intertrial speed $\left[F_{(8,168)}=5.3, p<0.0001\right]$, none. Vgat-StrDV-ChR2: Trial speed $\left[F_{(8,144)}=1.5\right.$, $p=0.15]$, none. Trial velocity $\left[F_{(8,144)}=7, p<\right.$ $0.0001], p<0.0035(40-66 \mathrm{~Hz})$. Intertrial speed $\left[F_{(8,144)}=2.3, p=0.02\right]$, none.

Similar to inhibiting $\mathrm{SNr}$ cells with Arch, inhibiting $\mathrm{SNr}$ cells by stimulating striatal fibers in $\mathrm{SNr}$ originating in dorsal or ventral portions of striatum is very effective at driving avoidance responses in the absence of an external ACS. However, when striatal fibers originating in both the dorsal and ventral striatum are excited in $\mathrm{SNr}$ at $\sim 40 \mathrm{~Hz}$, there is a strong suppression of avoidance responses, which is associated with impairments in directed movement to avoid (trial velocity), not in overall speed.

\section{Direct striatotegmental pathway activation in PPT blocks active avoidance responses}

Next, we expressed ChR2 in the NAc (Fig. 8A-C), which encompasses the ventromedial striatum. A

coursing via SNr into PPT, which fills PPT. The faint fluorescence in the cortex rostral to the accumbens, visible in Animal A, but not in Animal B, appears as dotted puncta at high magnification, which could be projection fibers from accumbens or faint expression in local GABAergic interneurons. C, 3D reconstructions showing the results of AAV injections to express ChR2 in GABAergic cells of NAc. The image is a placeholder for Movie 4. D. Example parasagittal section showing the tracks of an optical fiber implanted in PPT. 


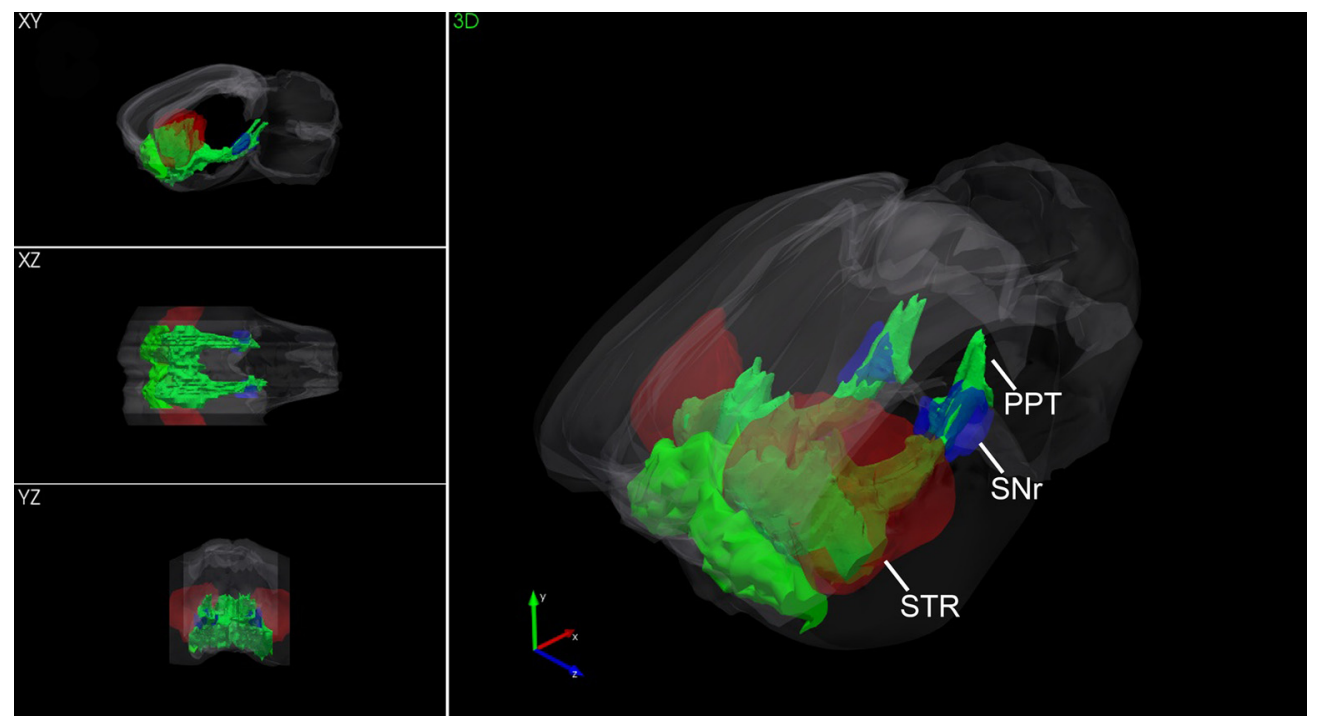

Movie 4. Video of the 3D reconstruction in Figure 8C. [View online]

single AAV injection was made in the NAc on each side (VgatAcb-ChR2). In these mice, we used in vitro (slice) and in vivo (anesthetized) recordings to confirm that $\mathrm{SNr}$ cells are inhibited by exciting with blue light ChR2-expressing GABAergic striatal fibers in $\mathrm{SNr}$ originating in the NAc (Fig. 9A-C). In slices, excitation of striatonigral fibers with trains of $1 \mathrm{~ms}$ pulses or continuous pulses of blue light produce strong IPSPs in the postsynaptic $\mathrm{SNr}$ neurons that robustly inhibit firing in a frequency-dependent manner (Fig. 9A). In vivo (anesthetized), this resulted in suppression of unit firing that was significant for continuous pulses and trains at $40 \mathrm{~Hz}$, but not at $66 \mathrm{~Hz}$ (Fig. 9B; $n=26$ cells; Tukey $t_{(88)}=4.6, p=0.01$ spontaneous vs $40 \mathrm{~Hz} ; t_{(88)}=6.3, p=0.0001$ spontaneous vs continuous), perhaps because of the level of IPSP adaptation present at the higher frequencies (consistent with Hormigo et al., 2019), but this issue was not explored further in the present study.

Examination of the histology from Vgat-Acb-ChR2 mice revealed that AAV injections in NAc produce, as expected, many fluorescent striatonigral fibers in $\mathrm{SNr}$ (Fig. $8 A-C$ ). However, many fibers originating in the NAc also continued to PPT, filling the ventrodorsal extent of PPT (Fig. $8 A-C$; see Fig. $8 A, B$, insets). To determine whether these fibers inhibit cells in PPT, we performed unit recordings in vivo (anesthetized) while applying blue light in the $\mathrm{SNr}$ of Vgat-Acb-ChR2 mice (Fig. 9C). This resulted in robust inhibition of PPT cells $(n=16)$ when blue light was applied at $10-66 \mathrm{~Hz}$, but not when blue light was applied as continuous pulses (Tukey $t_{(60)}=8.9, p<0.0001$ spontaneous vs $10 \mathrm{~Hz} ; t_{(60)}=8.1, p<0.0001$ spontaneous vs $40 \mathrm{~Hz} ; t_{(60)}=5.7$, $p=0.001$ spontaneous vs $66 \mathrm{~Hz}$ ). The cells inhibited by blue light were located in a strip matching the stereotaxic coordinate's location of PPT. Other cells recorded above, below, and within this strip of PPT were not affected by blue light in $\mathrm{SNr}(n=65)$. Thus, two striatal pathways are excited when blue light is applied in the $\mathrm{SNr}$ of Vgat-Acb-ChR2 mice: striatonigral fibers terminating in $\mathrm{SNr}$ and striatotegmental fibers terminating in PPT. Based on this knowledge, bilateral optical fibers were implanted in the $\mathrm{SNr}$ or PPT of Vgat-Acb-ChR2 mice (Fig. 3D [Movie 1]; Fig. $8 D$ ) to excite striatal fibers originating in the NAc. Using ACS + LCS trials, we tested the effect of stimulating the striatal fibers in $\mathrm{SNr}$ or PPT on signaled active avoidance driven by the ACS (Fig. 9D).

In ACS + LCS trials, excitation of GABAergic striatal fibers either in $\mathrm{SNr}$ (Fig. 9E, blue closed circles; 28 sessions in 4 mice) or
PPT (Fig. 9E, red open squares; 26 sessions in 4 mice) originating in the NAc produced an effect on active avoidance that resembled the effect of stimulating striatal fibers originating in the broader portion of striatum encompassing both the ventral and dorsal striatum (Vgat-StrDV-ChR2 mice in Fig. $7 C$, blue filled circles). Thus, blue light trains at $\sim 40 \mathrm{~Hz}$ strongly suppressed avoidance responses and response latency compared with ACS trials (Fig. 9E). The number of intertrial crossings increased but only for the intertrial intervals that follow escape responses (i.e., trials with blue light trains at $\sim 40 \mathrm{~Hz}$ in which avoidance failed), typical of animals that were just shocked to escape, as we previously noted. The main repeated-measures factor of the mixed ANOVA [ $F$ and $p$ values] and the Tukey test $p$ values for ACS + LCS trials that are significantly different compared with ACS trials are indicated here for each measure per group. Vgat-Acb-ChR2 $\rightarrow \mathrm{SNr}$ : Avoids $\left[F_{(8,240)}=43.5, p<0.0001\right], p<0.00,001(40-66 \mathrm{~Hz})$. Latency $\left[F_{(8,240)}=37.9, p<0.0001\right], p<0.0008(20-100 \mathrm{~Hz})$. Intertrial crossings $\left[F_{(8,240)}=9.7, p<0.0001\right], p<0.0001(20$ $66 \mathrm{~Hz}$ and continuous). Vgat-Acb-ChR2 $\rightarrow$ PPT: Avoids $\left[F_{(8,176)}=\right.$ 62.4, $p<0.0001], p=0.001(20 \mathrm{~Hz}), p<0.0001(40-66 \mathrm{~Hz})$. Latency $\left[F_{(8,176)}=61.5, p<0.0001\right], p<0.00001(20-100 \mathrm{~Hz})$. Intertrial crossings $\left[F_{(8,176)}=28.8, p<0.0001\right], p<0.0001(20$ $66 \mathrm{~Hz})$.

Video tracking showed that the block of avoidance responses during ACS + LCS trials at 40-66 Hz in SNr was not accompanied by a reduction in trial speed but exhibited a reduction in trial velocity (Fig. 9E), indicating that animals moved at control (ACS trial) speeds but not in the direction of the safe compartment. However, exciting striatotegmental fibers in PPT modestly suppressed trial speed and velocity for all blue light trains, but not for continuous blue light, indicating that the animals moved less overall during the trial. In general, for trials in which avoidance responses were blocked, there was an increase in intertrial speed consistent with the increase in intertrial crossings for these trials. Interestingly, continuous blue light in $\mathrm{SNr}$, but not in PPT, slightly increased trial speed and intertrial speed, but not trial velocity. This effect may be because of the distinct effects on $\mathrm{SNr}$ cells produced by activation of striatonigral fibers with continuous blue light compared with trains (Fig. 9A-C) and deserves further investigation in future work. The main repeated-measures factor of the mixed ANOVA [ $F$ and $p$ values] and the Tukey 
A

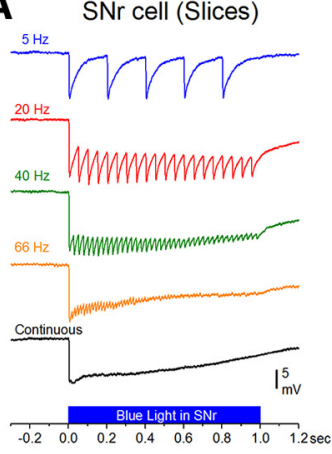

B

SNr units (in vivo)

C
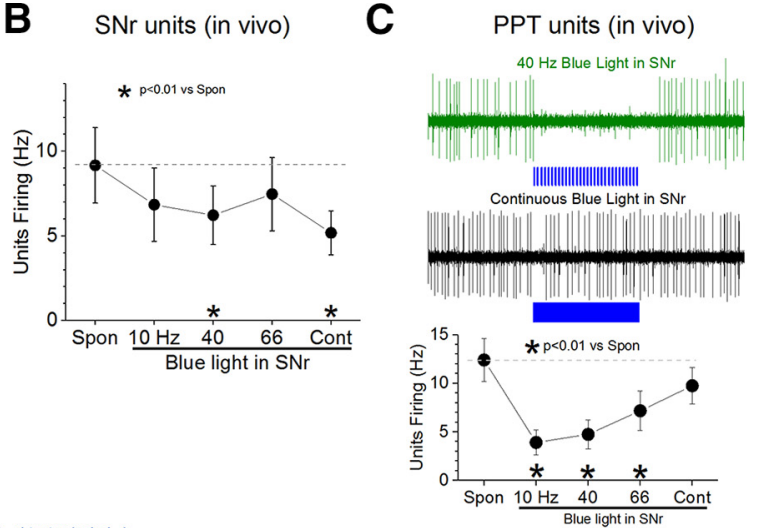

D

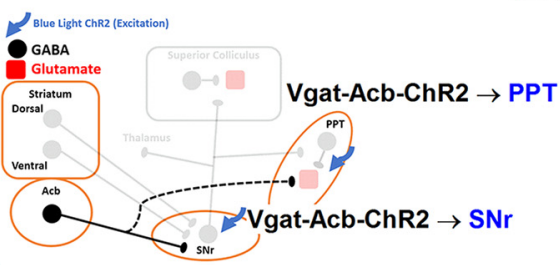

E

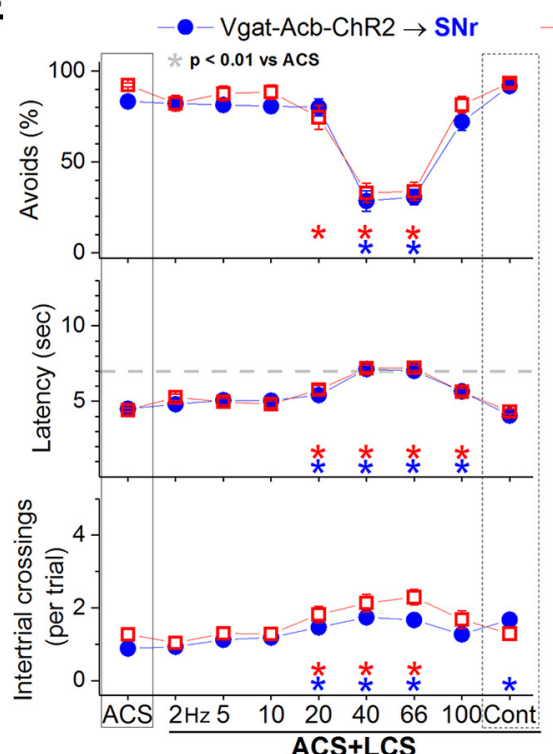

- - Vgat-Acb-ChR2 $\rightarrow$ PPT
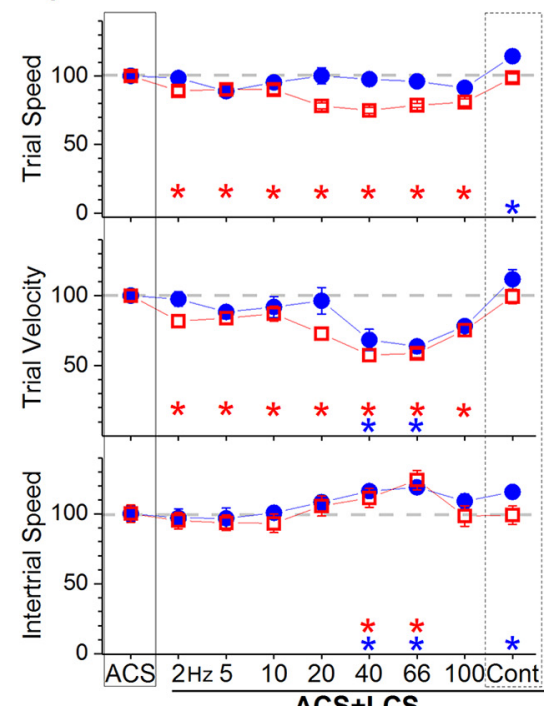

Figure 9. Effect of NAC pathway activation in SNr or PPT on signaled active avoidance responses. $\boldsymbol{A}$, Example IPSPs evoked in an $\mathrm{SNr}$ cell (whole-cell recording) from a Vgat-Acb-ChR2 mouse slice by exciting GABAergic fibers originating in NAc cells with blue light. Each trace is the average of 6 stimulus trials and reflects different patterns of blue light stimulation, including trains of $1 \mathrm{~ms}$ pulses (at 5, 20,40, and $66 \mathrm{~Hz}$ ) and continuous pulses delivered for $1 \mathrm{~s}$. B, Population data showing the effect of exciting GABAergic fibers of NAc cells in $\mathrm{SNr}$ with blue light on the firing of $\mathrm{SNr}$ units $(n=26)$ recorded in isoflurane-anesthetized Vgat-Acb-ChR2 mice. Plot represents the spontaneous firing of $\mathrm{SNr}$ cells compared with different patterns of blue light simulation, including trains of $1 \mathrm{~ms}$ pulses $(10,40$, and $66 \mathrm{~Hz})$ and continuous pulses delivered for $1 \mathrm{~s}$. C, Blue light applied in SNr of Vgat-AcbChR2 mice inhibits cells in PPT, but there is much less inhibition when continuous light is used. Top, Example PPT unit activity showing the effect of a train $(40 \mathrm{~Hz})$ and a continuous pulse $(1 \mathrm{~s})$ of blue light. Note the robust inhibition in response to the train but not the continuous pulse. Bottom, Population data comparing spontaneous PPT cell $(n=16)$ firing versus different patterns of blue light simulation applied in $\mathrm{SNr}$, including trains of 1 ms pulses $(10,40$, and $66 \mathrm{~Hz})$ and continuous pulses delivered for 1 S. D. Schematic of the pathways activated in these experiments, which includes striatonigral and striatotegmental fibers that originate in the NAc. $\boldsymbol{E}$, Effect of blue light applied in the SNr (blue closed circles) or the PPT (red open squares) on ACS +LCS trials for animals that express ChR2 in GABAergic fibers that originate in the NAc (Vgat-Acb-ChR2; 26-28 sessions in 4 mice/group). Note the strong inhibition of avoidance responses for blue light trains at 40-66 Hz delivered in either SNr or PPT, indicating that excitation of GABAergic striatotegmental fibers that originate in the $\mathrm{NAC}$ and course through $\mathrm{SNr}$ block signaled active avoidance. Right, Trial speed, trial velocity, and intertrial speed for the data in the left panel. test $p$ values for ACS + LCS trials that are different compared with ACS trials are indicated here for each measure per group. Vgat-Acb-ChR2 $\rightarrow$ SNr: Trial speed $\left[F_{(8,232)}\right.$ $=7.4, p<0.0001], p=0.004$ (continuous). Trial velocity $\left[F_{(8,232)}=12.8, p<0.0001\right]$, $p<0.0001 \quad(40-66 \mathrm{~Hz})$. Intertrial speed $\left[F_{(8,232)}=16.1, p<0.0001\right], p<0.0001(40-$ $66 \mathrm{~Hz}$ ), $p=0.00,006$ (continuous). VgatAcb-ChR2 $\rightarrow$ PPT: Trial speed $\left[F_{(8,176)}=\right.$ 26.8, $p<0.0001], p<0.003(2-100 \mathrm{~Hz})$. Trial velocity $\left[F_{(8,176)}=43.8, p<0.0001\right]$, $p<0.003 \quad(2-100 \mathrm{~Hz})$. Intertrial speed $\left[F_{(8,176)}=26.1, p<0.0001\right], p=0.01(40 \mathrm{~Hz})$, $p<0.00001(66 \mathrm{~Hz})$.

Together, our results indicate that the primary reason why avoidance responses are suppressed by stimulating striatal fibers in $\mathrm{SNr}$ at $40-66 \mathrm{~Hz}$ is that these frequencies activate striatal fibers on their way to PPT. Indeed, direct inhibition of SNr cells with Arch, which does not excite any fibers coursing through $\mathrm{SNr}$ to PPT, very effectively drives avoidance responses. The poor inhibition of $\mathrm{SNr}$ cells by striatal fiber activation in $\mathrm{SNr}$ at $\sim 66 \mathrm{~Hz}$ in vivo (Fig. 9B) may also contribute to the poor ability to drive avoidance responses at this frequency. Moreover, the finding that blue light applied in $\mathrm{SNr}$ as trains at $\sim 40 \mathrm{~Hz}$, but not as continuous pulses, inhibits cells in PPT (Fig. 9C) can explain the selectivity of the suppression of avoidance responses when light is applied at $\sim 40 \mathrm{~Hz}$. Thus, striatal GABAergic cells can drive avoidance responses by inhibiting $\mathrm{SNr}$ cells and block avoidance responses by inhibiting PPT cells. The fact that selective stimulation of GABAergic striatotegmental fibers in PPT abolishes active avoidance responses (Fig. 9E, red open squares) underscores the critical role of PPT in the expression of signaled active avoidance, which we previously demonstrated (Hormigo et al., 2019).

\section{Precluding SNr deactivation does not impair active avoidance expression} Optogenetic excitation of GABAergic afferents in PPT that originate in $\mathrm{SNr}$ (Hormigo et al., 2019), zona incerta (Hormigo et al., 2020), or striatum (present study) abolish signaled active avoidance responses to an ACS, while sparing escape responses to the foot-shock. Whereas PPT is essential for the expression of signaled active avoidance behavior, the role of GABAergic afferents in PPT that can abolish the behavior when excited, including those from $\mathrm{SNr}$, is not clear. Optogenetic inhibition of SNr GABAergic cells during the avoidance interval is a highly effective CS to drive avoidance responses. In addition, the firing of $\mathrm{SNr}$ cells changes during 
A

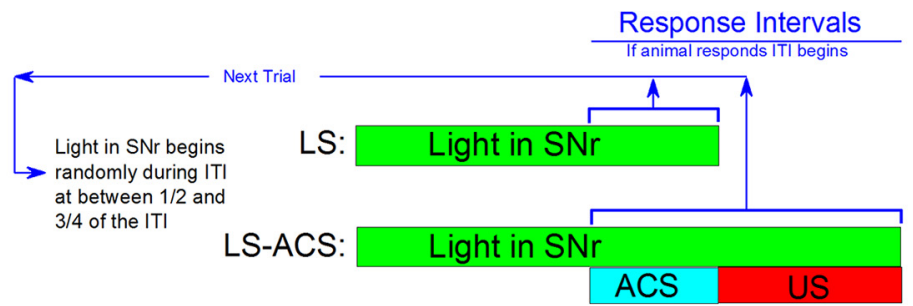

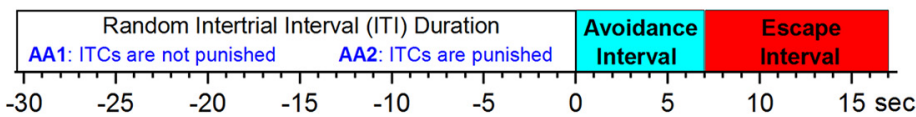

B

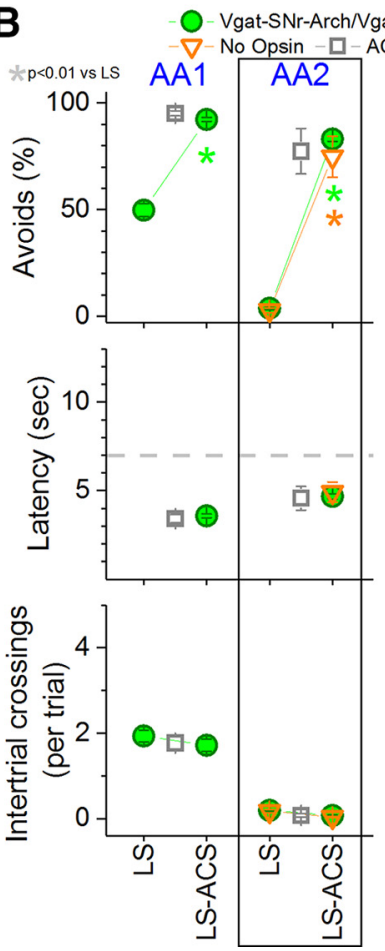
(No Light)

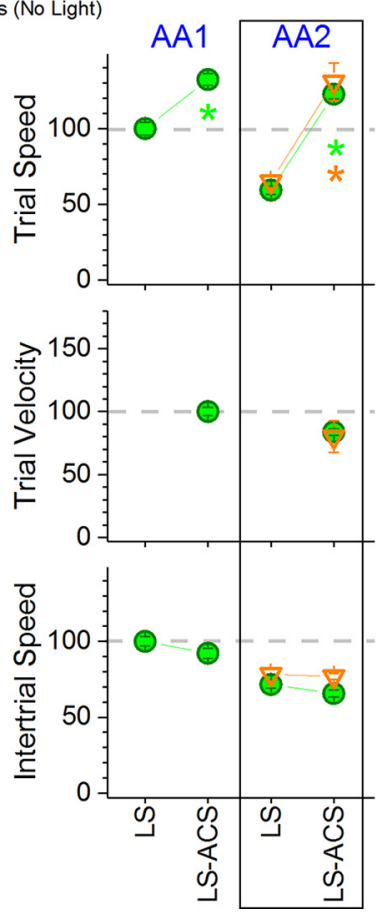

C - O-Vgat-SN-ArchNaat-SN-IC++ $-\nabla$ - No Opsin - $\square$-ACS trials (No Light)
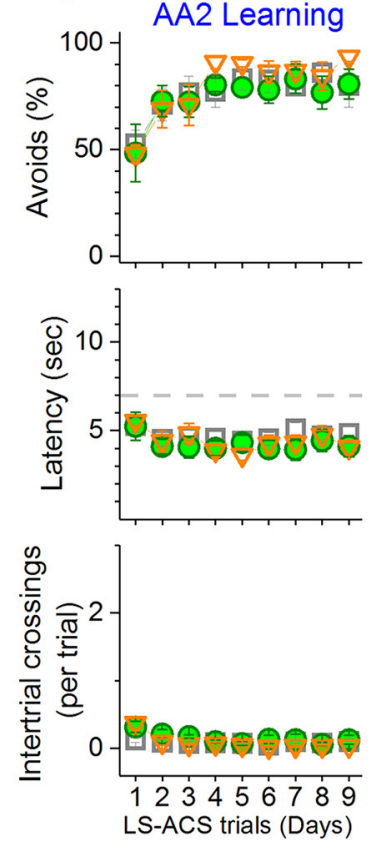

Figure 10. Effects of precluding SNr deactivation on signaled active avoidance. A, Schematic detailing LS and LS-ACS trials. LS trials are No CS trials in which optogenetic light begins randomly during the preceding intertrial interval and continues during the unsignaled avoidance interval. LS-ACS trials are ACS trials in which optogenetic light begins randomly during the preceding intertrial interval and continues during the avoidance and escape intervals (until the animal avoids or escapes). The optogenetic light applied in the SNr of Vgat-SNr-Arch (green light) and of Vgat-SNr-IC+ + (blue light) mice is used to inhibit cells during the intertrial interval to preclude the SNr deactivation that occurs when animals actively avoid during the avoidance interval. Shuttling during the intertrial interval (with or without optogenetic light) are intertrial crossings, which in AA2 procedures are punished, requiring passive avoidance during the intertrial interval. $\boldsymbol{B}$, Effect of precluding SNr deactivation in GABAergic SNr cells on signaled active avoidance. The results from Vgat-SNr-Arch and Vgat-SNr-IC ++ animals were combined into a single group $(n=8)$ since the effects did not differ significantly. Right panels, Trial speed, trial velocity, and intertrial speed for the data in the left panels. No Opsin animals (open triangles) subjected to the same procedures are also shown $(n=6)$. Precluding SNr deactivation did not impair signaled active avoidance performance; the percentage of avoids was normal in LS-ACS trials and much higher than in LS trials for both AA1 and AA2 procedures. C, Effect of LS-ACS trials in naive mice to determine whether precluding SNr deactivation impairs learning of signaled active avoidance (AA2 procedure). Naive Vgat-SNr-Arch and Vgat-SNr-IC++ were trained using only LS-ACS trials $(n=6)$, and an additional group of mice $(n=6)$ trained only with ACS trials (No light). Precluding SNr deactivation did not impair learning of signaled active avoidance.

active avoidance responses, including robust deactivation of a large proportion of cells. This raises the question of whether the deactivation of SNr firing observed during avoidance responses must occur to express an avoidance responses. For instance, deactivation of $\mathrm{SNr}$ firing could be driving the expression of active avoidance responses by disinhibiting (exciting) PPT, which drive avoidance responses (Hormigo et al., 2019). Alternatively, SNr firing may have a permissive role in the expression of active avoidance. In this alternative scenario, $\mathrm{SNr}$ activation (including abnormal activation) can block (gate) the expression of avoidance responses, but $\mathrm{SNr}$ deactivation does not normally drive these responses, it simply assures expression can occur.

To investigate these options, we tested whether precluding $\mathrm{SNr}$ deactivation would impair signaled active avoidance. To preclude the $\mathrm{SNr}$ deactivation that occurs during avoidance responses (Fig. 2), we inhibited SNr cells before the onset of the avoidance interval at a random time beginning around onefourth of the preceding intertrial interval; the inhibition continued during the avoidance/escape intervals, as in ACS+LCS trials (Fig. 10A). Thus, SNr is already inhibited at the onset of the ACS, precluding the natural SNr deactivation that occurs during avoidance responses. In addition, because of the extended presentation of the optogenetic light on each trial in these experiments, we used two groups of animals, Vgat-SNr-Arch $(n=5)$ and Vgat-SNr-IC $++(n=3)$ mice, that differ in the optogenetic light wavelength and power used to inhibit $\mathrm{SNr}$ cells $(3-6 \mathrm{~mW}$ blue light for Vgat-SNr-IC ++ and $25-35 \mathrm{~mW}$ green light for Vgat-SNr-Arch). These animals were combined into a single 
group ( $n=8)$ after determining that there were no significant differences in the results. An additional group of No Opsin mice was subjected to the same procedures $(n=6)$. Three types of trials were presented randomly: LS, LS-ACS, and ACS trials (Fig. $10 A, B)$. In LS trials, the optogenetic precluding light began before a No CS trial. In LS-ACS trials, the optogenetic precluding light began before an ACS trial. Thus, the only difference between LS trials and LS-ACS trials is the presence of the ACS. In addition, we tested control ACS trials (without optogenetic light). The mice were first tested for active avoidance with the $\mathrm{AA} 1$ procedure, and later in the AA2 procedure. In AA2, mice must passively avoid during the intertrial interval, and therefore the intertrial crossings do not confound the number of true avoidance responses.

If the $\mathrm{SNr}$ deactivation that occurs during the signaled avoidance interval (Fig. 2) is important to drive avoidance responses, precluding the $\mathrm{SNr}$ deactivation should impair active avoidance in LS-ACS trials. Instead, our results show that precluding $\mathrm{SNr}$ deactivation does not impair the ability of the mice to perform signaled active avoidance. During the AA1 procedure, mice displayed normal active avoidance performance in LS-ACS trials, including response latencies and number of intertrial crossings similar to ACS trials (Fig. 10B; Tukey, AA1 Avoids, $t_{(59)}=24.6$, $p<0.0001$ LS vs LS-ACS). However, these mice also performed large numbers of spurious avoidance responses during LS trials ( $\sim 50 \%)$, which are not signaled by the ACS. This is likely a consequence of the elevated motor activity displayed by these animals because of the extended $\mathrm{SNr}$ inhibition. Therefore, the animals were subjected to the AA2 procedure, which punishes intertrial crossings, to determine whether they are able to inhibit their intertrial crossings by passively avoiding during the intertrial interval while maintaining active avoidance responses to the ACS. Indeed, during the AA2 procedure, mice eliminated their intertrial crossings as well as their spurious active avoidance responses during LS trials (Fig. 10B). This is consistent with the fact that only the ACS predicts the US in these trials, and therefore should be the only stimulus consistently driving avoidance (shuttling) responses. In AA2, trial and intertrial speed during LS trials was reduced by $30 \%-50 \%$ compared with the AA1 procedure, as the animals reduced their spontaneous motor activity in the AA2 procedure. Moreover, trial speed and velocity during LS-ACS trials continued to be elevated during AA2, as the animals continued to effectively avoid (Tukey, AA2 Avoids, $t_{(71)}=$ $60.1, p<0.0001$, LS vs LS-ACS), while intertrial speed was reduced to levels equivalent to LS trials, as the animals mostly moved to avoid during the avoidance interval of LS-ACS trials. Importantly, in the AA2 procedure, these animals continued to actively avoid in LS-ACS trials at levels that were not different from control ACS trials. Similar results were observed in No Opsin mice subjected to AA2 procedures, indicating that precluding SNr deactivation has little effect on active avoidance (Fig. 10B). The results indicate that SNr modulation during the avoidance interval does not drive signaled active avoidance responses, and supports the contention that $\mathrm{SNr}$ has a permissive role in the expression of signaled active avoidance.

\section{Precluding SNr deactivation does not impair active avoidance learning}

An important consideration is that, when animals are well trained in signaled active avoidance (habitual responding), the role of $\mathrm{SNr}$ is less important than when the behavior is incipient because of differences between goal-directed and habitual responding (Yin and Knowlton, 2006; O’Hare et al., 2016). If this is the case, precluding $\mathrm{SNr}$ deactivation during the learning phase should interfere with the ability of animals to learn. To test this possibility, we trained three groups of naive mice in signaled active avoidance using the AA2 procedure to eliminate spurious avoidance responses because of intertrial crossings. One group consisted of Vgat-SNr-Arch and Vgat-SNr-IC + + mice combined together $(n=6 ; 3$ of each), and a second group included No Opsin controls $(n=6)$. Both of these groups had bilateral optical fibers in SNr. A third group of naive mice were trained with regular ACS trials, in the absence of light stimulation $(n=8)$. Thus, we precluded SNr deactivation by presenting LS-ACS trials starting from the first trial and throughout nine daily learning sessions. We found that there was no difference in the avoidance rate, response latency, and number of intertrial crossings between these three groups (Fig. 10C). These results indicate that precluding SNr deactivation does not impair the ability to learn signaled active avoidance driven by the ACS. Taken with our previous findings, these results indicate that $\mathrm{SNr}$ deactivation does not drive signaled active avoidance responses. This finding is compatible with our alternative hypothesis that $\mathrm{SNr}$ firing has a permissive role in active avoidance responses.

\section{Modulation of GABAergic cell firing in striatum, NAc, and SNr with DREADDs has modest effects on signaled active avoidance}

We used DREADDs to supplement the optogenetics experiments. In contrast to optogenetics, DREADDs modulate neural activity in a time-unselective manner when treated with CNO. Figure $11 \mathrm{~A}$ shows sample histologic sections from an animal expressing $\mathrm{hM} 4 \mathrm{D}(\mathrm{Gi})$ in GABAergic $\mathrm{SNr}$ cells after an $\mathrm{AAV}$ injection.

Excitation (Vgat-Str-hM3Dq, $n=6$ mice) or inhibition (VgatStr-hM4Di, $n=6$ mice) of GABAergic cells in striatum (involving the same dorsal and ventral striatum injections sites used for optogenetics) had only modest effects on signaled active avoidance (Fig. $11 B$ ). There was a small $(<7 \%)$ suppression of the percentage of avoidance responses, and a small increase $(\sim 0.4 \mathrm{~s})$ in response latency. However, these effects were similar for exciting or inhibiting striatum GABAergic cells, indicating that they may be nonspecific effects of broad manipulations of striatum activity. More selectively, the number of intertrial crossings (AA1 procedure) were reduced but only modestly $(\sim 23 \%)$ when striatum GABAergic cells were inhibited (Fig. 11B). Thus, modulating the activity of GABAergic striatum cells with DREADDs has only modest effects on signaled active avoidance.

Excitation (Vgat-Acb-hM3Dq, $n=6$ mice) or inhibition (Vgat-Acb-hM4Di, $n=6$ mice) of GABAergic cells in the NAc did not significantly impair signaled active avoidance or response latency in the AA1 procedure (Fig. 11C). However, excitation of GABAergic cells in the NAc robustly increased the number of intertrial crossings in the AA1 procedure (Tukey, $t_{(43)}=9.3$, $p<0.0001)$. This led to testing these animals in the AA2 procedure (which punishes intertrial crossings, requiring passive avoidance during the intertrial interval). During AA2, exciting NAc GABAergic cells modestly suppressed $(<7 \%)$ the number of avoidance responses to the ACS without affecting response latency. In addition, while the number of intertrial crossings was virtually nil during AA2, exciting NAc GABAergic cells modestly increased those responses (Tukey $t_{(30)}=4.5, p=0.002$ ), which represent passive avoidance failures. Therefore, in AA1, exciting NAc GABAergic firing increases spontaneous intertrial crossings and, in AA2, modestly impairs active avoidance to the ACS and passive avoidance during the intertrial interval. 

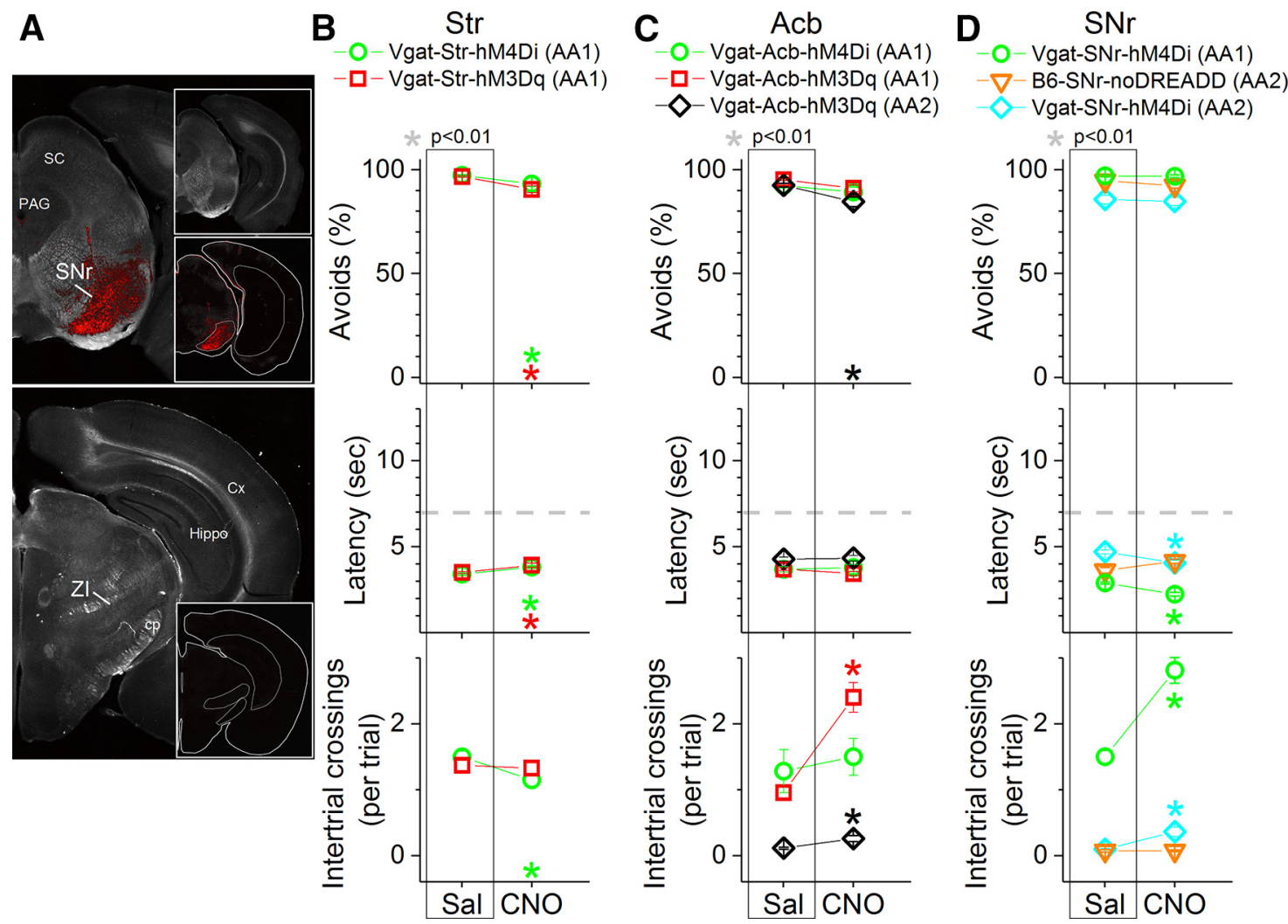

Figure 11. Effects of modulating (exciting or inhibiting) striatal, NAC, or SNr cells with DREADDs on signaled active avoidance. $A$, Example coronal sections showing mCherry fluorescence after an AAV injection in the SNr of a Vgat-SNr-hM4Di mouse. Top, Centered on SNr. Bottom, The zona incerta located more rostral, which is devoid of fluorescence. The main images blend a dark-field image of the section with the red channel of the $\mathrm{mCherry}$ fluorescence image. Insets, The dark-field (top) and the fluorescence image (bottom) alone. (P, Cerebral peduncle. $\boldsymbol{B}-\boldsymbol{D}$, Comparison of the effects of saline (Sal) and CNO on ACS trials in mice that express hM4D(Gi) or hM3D(Gq) to inhibit or excite, respectively, GABAergic neurons in broad areas of striatum, including dorsal and ventral striatum $(\boldsymbol{B}), \mathrm{NAC}(\boldsymbol{C})$, and $\mathrm{SNr}(\boldsymbol{D})$. All animals are subjected to AA1 procedures, and subsequently to $\mathrm{AA2}$ procedures if the number of intertrial crossings was increased by CNO during AA1. Excitation of GABAergic cells in the NAc (not in dorsal or ventral striatum), or inhibition of GABAergic cells in SNr, increased the number of intertrial crossings during AA1 procedures. In AA2, these manipulations produced modest failures in passive avoidance with negligible effects on signaled active avoidance $(n=6 /$ group). $* p<0.01$, Sal versus $\mathrm{CNO}$ (Tukey).

Finally, we tested the effects of modulating SNr GABAergic cells with DREADDs. The effects of excitation and inhibition of SNr GABAergic cells with DREADDs have been previously tested in the AA1 procedure (Hormigo et al., 2016). Exciting SNr GABAergic cells with DREADDs robustly impairs signaled active avoidance in the AAl procedure, mimicking the effects of optogenetic excitation (Hormigo et al., 2016). Conversely, in the present study, we found that inhibiting SNr GABAergic cells (AA1 procedure) produces a robust increase in intertrial crossings (Vgat-SNr-hM4Di (AA1), $n=6$ mice, Fig. $11 D$; Tukey $t_{(42)}$ $=10.9, p<0.0001)$ that is complementary to exciting NAc GABAergic cells (Fig. 11C). Therefore, based on the increase in intertrial crossings in $\mathrm{AAl}$, we tested the effect of inhibiting $\mathrm{SNr}$ GABAergic cells in the AA2 procedure (Vgat-SNr-hM4Di (AA2), $n=6$ mice; Fig. $11 D$ ). The number of intertrial crossings was virtually nil during $\mathrm{AA} 2$, and similar to exciting GABAergic cells in NAc, inhibiting GABAergic cells in $\mathrm{SNr}$ modestly increased the number of intertrial crossings, which represents an impairment in passive avoidance (Fig. $11 D$; Tukey $t_{(78)}=9.14$, $p<0.0001)$. This occurred without impairing active avoidance driven by the ACS. In addition, during either AA1 or AA2, inhibiting SNr GABAergic cells leads to faster response latencies.

Since the effects of inhibiting SNr GABAergic cells on intertrial crossing during $\mathrm{AA} 2$ are rather modest, as a control, we injected the same cre-AAV into the SNr of non-cre C57/BL6 mice (B6-SNr-noDREADD, $n=6$ mice), and tested the effect of
$\mathrm{CNO}$ in the AA2 procedure (Fig. 11D). This resulted in no significant effects, including no increase in the number of intertrial crossings, which highlights the significance of the failure in passive avoidance when SNr GABAergic cells are inhibited. In conclusion, inhibiting GABAergic SNr cells selectively impairs passive avoidance in the AA2 procedure, albeit modestly.

\section{Inhibition of GABAergic afferents in PPT modulates, but} does not block, signaled active avoidance

The previous results indicate that the $\mathrm{SNr}$ deactivation observed during active avoidance responses is not required for the expression of avoidance responses, and that inhibiting GABAergic cells in striatum, $\mathrm{NAc}$, or $\mathrm{SNr}$ does not impair signaled active avoidance. Perhaps, apart from SNr, other GABAergic nuclei that project to PPT are also simultaneously deactivated and their inhibition drives active avoidance responses by disinhibiting PPT. Indeed, zona incerta GABAergic neurons have been recently shown to have a role similar to $\mathrm{SNr}$ in controlling signaled active avoidance responses through their projections to PPT (Hormigo et al., 2020). To determine whether GABAergic $\mathrm{PPT}$ afferents, originating in $\mathrm{SNr}$ and zona incerta, are required for signaled active avoidance, we bilaterally injected a Cre-inducible AAV into both the $\mathrm{SNr}$ and the zona incerta of Vgat-cre mice [AAV5-EF1a-DIO-eArch3.0-EYFP; UNC Vector Core] (Vgat-SNZI-Arch). These animals were implanted with bilateral optical fibers in the PPT to inhibit GABAergic fibers originating 

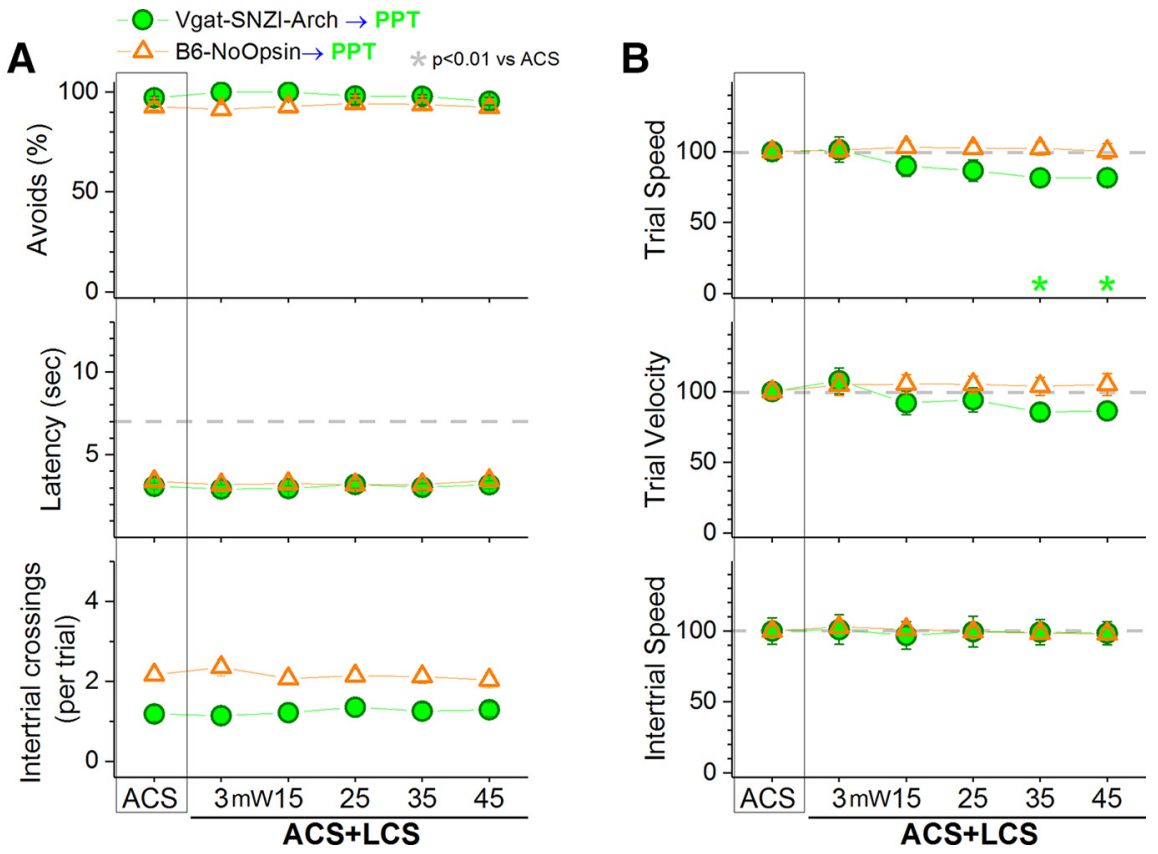

Figure 12. Effects of inhibiting PPT GABAergic afferents that originate in SNr and zona incerta on signaled active avoidance. $\boldsymbol{A}$, Effect of green light applied in the PPT on ACS + LCS trials for animals that express Arch in GABAergic fibers originating in SNr and zona incerta (Vgat-SNZI-Arch; $n=6$ ). This did not impair active avoidance. The same procedures were applied to a group of No Opsin mice (B6-No Opsin; $n=6$ ). $\boldsymbol{B}$, Trial speed, trial velocity, and intertrial speed for the data in $\boldsymbol{A}$. Inhibiting GABAergic afferents in PPT suppressed trial speed (at the higher green light powers) only in the Vgat-SNZI-Arch mice

in $\mathrm{SNr}$ and zona incerta. Arch can inhibit presynaptic neurotransmitter release reducing the effects of these afferents in PPT (Wiegert and Oertner, 2016; Wiegert et al., 2017).

In ACS + LCS trials (AA1), inhibition of GABAergic fibers in the PPT that originate in the SNr and zona incerta did not suppress the ability of the animals to perform signaled active avoidance (Fig. 12A). There was no impairment in the number of avoidance responses or any other task parameter. The main repeated-measures factor of the mixed ANOVA $[F$ and $p$ values] and the Tukey test $p$ values for ACS + LCS trials that are significantly different compared with ACS trials are indicated here for each measure per group. Vgat-SNZI-Arch $\rightarrow$ PPT: Avoids $\left[F_{(5,60)}=4.09, p=0.002\right]$, none. Latency $\left[F_{(5,60)}=1.5\right.$, $p=0.2]$, none. Intertrial crossings $\left[F_{(5,60)}=1, p=0.4\right]$, none. B6No Opsin $\rightarrow$ PPT: Avoids $\left[F_{(5,216)}=1, p=0.4\right]$, none. Latency $\left[F_{(5,216)}=1.1, p=0.3\right]$, none. Intertrial crossings $\left[F_{(5,216)}=1.4\right.$, $p=0.2]$, none.

Video tracking revealed a suppression of trial speed at the highest green light intensities used, but no suppression of trial velocity or intertrial speed (Fig. 12B). Thus, inhibition of GABAergic afferents in PPT (known to impair signaled active avoidance when excited) does not impair signaled active avoidance. However, inhibition of these afferents has an effect on trial speed, which is consistent with a role of GABAergic afferents in modulating (but not in driving) the movement during avoidance responses. The effects on trial speed were absent in a group of C57/B6 mice subjected to the same optogenetic procedures but that did not express Arch (B6-NoOpsin; Fig. 12A,B). The main repeated-measures factor of the mixed ANOVA [ $F$ and $p$ values] and the Tukey test $p$ values for ACS + LCS trials that are different compared with ACS trials are indicated here for each measure per group. Vgat-SNZI-Arch $\rightarrow$ PPT: Trial speed $\left[F_{(5,60)}=6.7\right.$, $p<0.0001], p<0.0001(35-45 \mathrm{~mW})$. Trial velocity $\left[F_{(5,60)}=3.7\right.$, $p=0.005]$, none. Intertrial speed $\left[F_{(5,60)}=\right.$ $0.2, p=0.9$ ], none. B6-No Opsin $\rightarrow$ PPT: Trial speed $\left[F_{(5,216)}=0.4, p=0.8\right]$, none. Trial velocity $\left[F_{(5,216)}=0.2, p=0.9\right]$, none. Intertrial speed $\left[F_{(5,216)}=0.8\right.$, $p=0.5]$, none.

We conclude that the $\mathrm{SNr}$ and zona incerta GABAergic pathways reaching PPT do not drive active avoidance responses; and since their excitation blocks avoidance responses, these afferents have a permissive role in the expression of signaled active avoidance.

\section{Discussion}

The purpose of this study was to investigate the role of the main output of the basal ganglia through $\mathrm{SNr}$ on a signaled locomotor action, known as signaled active avoidance, by which mice move away during a warning signal to avoid a threat. We found that optogenetic inhibition of $\mathrm{SNr}$ cells serves as an effective CS to drive avoidance responses by disinhibiting target structures that are essential for signaled active avoidance, such as PPT. Single-unit recordings in animals performing signaled active avoidance showed that many $\mathrm{SNr}$ cells deactivated, whereas others activated or were unaffected, during the expression of avoidance responses. We tested whether $\mathrm{SNr}$ deactivation was driving the avoidance response by precluding $\mathrm{SNr}$ deactivation or inhibiting $\mathrm{SNr}$ fibers in PPT. Our results show that interfering with $\mathrm{SNr}$ modulation does not impair signaled active avoidance, indicating that $\mathrm{SNr}$ modulation does not drive active avoidance responses. Moreover, activation of basal ganglia output in PPT by solely exciting striatotegmental fibers is sufficient to abolish active avoidance responses, but it is uncertain when this may happen other than during pathology. Our results indicate that $\mathrm{SNr}$ has a permissive gating role in signaled active avoidance, by which deactivation allows, but does not drive, response expression. $\mathrm{SNr}$ output may normally provide downstream structures, which are essential for expression of signaled active avoidance, information about the ongoing movement so those structures can regulate the speed or other movement variables of the avoidance response.

\section{SNr firing and active avoidance}

$\mathrm{SNr}$ cells pause their tonic firing during orienting movements (Hikosaka and Wurtz, 1983; Wurtz and Hikosaka, 1986; Schmidt et al., 2013). Our data indicate that, during active avoidance, the firing of $\mathrm{SNr}$ cells relates to the movement of the animal. SNr cells do not sense the CS signal that drive the avoidance response; $\mathrm{SNr}$ firing does not change robustly during the CS presentation in the absence of movement. Instead, $\mathrm{SNr}$ cells appear to monitor the avoidance response as it occurs. Our findings are in good agreement with previous studies showing that $\mathrm{SNr}$ cells continuously code movement variables (Fan et al., 2012; Barter et al., 2015; Yin, 2017). Moreover, their inhibitory afferents, striatal cells, fire mainly during movement (Cui et al., 2013; Markowitz et al., 2018; Sales-Carbonell et al., 2018), most likely as a consequence of their sensitivity to somatosensory input 
(Carelli and West, 1991; Robbe, 2018). Thus, SNr cells supply target structures, like PPT, with information about the ongoing movement during expression of the avoidance response. Intriguingly, cross-correlation analyses indicate that the $\mathrm{SNr}$ cells that deactivate during movement code avoidance speed more sharply than general movement speed. Further investigation will need to determine whether this relates to features of the movement (e.g., faster during avoidance vs general moment) or its purpose (e.g., goaldirected vs spontaneous movement).

\section{SNr deactivation and active avoidance}

Most of the recorded SNr cells deactivated during the expression of active avoidance responses. In addition, broad optogenetic inhibition of $\mathrm{SNr}$ cells serves as a very effective CS to drive either active or passive avoidance responses. The ability of SNr inhibition to drive avoidance responses requires learning, as it does not occur innately in naive mice. These findings could imply that, during active avoidance, the external CS deactivates SNr cells. However, as noted, $\mathrm{SNr}$ deactivation during active avoidance responses closely relates to the avoidance movement, not the CS presentation driving the movement. This raises questions about why optogenetic SNr inhibition is as effective as a natural CS to drive avoidance responses and what is the purpose of $\mathrm{SNr}$ cell deactivation measured during active avoidance.

Regarding the effectiveness of optogenetic SNr inhibition to serve as a CS, we found that this disinhibits the targets of $\mathrm{SNr}$ that are important for expressing active avoidance responses, effectively exciting cells in PPT (Fig. $4 E$ ) and superior colliculus (Hormigo et al., 2016). Previously, we found that direct excitation of either of these two target structures serves as a very effective CS to drive avoidance responses in the absence of a natural CS (Hormigo et al., 2019), and stronger excitation can also drive innate escape responses (Brandao et al., 1994; Shang et al., 2015; Caggiano et al., 2018; Hormigo et al., 2019). Thus, the neural activity disinhibited in PPT and/or superior colliculus during $\mathrm{SNr}$ inhibition becomes conditioned to drive avoidance responses, substituting for the natural sensory CS that normally drives these responses. In other words, optogenetic $\mathrm{SNr}$ inhibition is akin to exciting its downstream midbrain targets, both of which (when directly excited) can be conditioned to drive avoidance responses in the absence of a natural external CS.

Regarding the purpose of SNr cell deactivation measured during active avoidance, we found that precluding $\mathrm{SNr}$ deactivation occurring during the avoidance movement does not impair signaled active avoidance, indicating that $\mathrm{SNr}$ deactivation does not drive avoidance responses. We also explored the possibility that this finding is the result of using well-trained animals, since overtrained habitual responding in rodents becomes less dependent on the basal ganglia (Yin and Knowlton, 2006; Yin et al., 2009). However, precluding SNr deactivation during the learning phase did not impair acquisition of signaled active avoidance either.

Our results show that $\mathrm{SNr}$ inhibition, with optogenetics or DREADDs, increases the number of spurious crossings during the intertrial interval, suggesting that $\mathrm{SNr}$ deactivation may impair their ability to inhibit spontaneous behavior. In traditional (unsignaled) passive avoidance procedures, animals must learn to suppress their tendency to perform certain spontaneous behaviors to avoid a harmful consequence. Thus, we trained animals using a signaled active avoidance procedure (AA2) that simultaneously tests passive avoidance. During $\mathrm{SNr}$ inhibition, mice are able to passively avoid harmful consequences (inhibit their tendency to produce spurious crossings during the intertrial interval), albeit with significantly more failures than when $\mathrm{SNr}$ is not inhibited. Thus, while SNr inhibition does not impair signaled active avoidance, it does slightly, but consistently, impair the ability of mice to perform passive avoidance. Future work should further clarify the role of these circuits in passive avoidance.

\section{What is the role of $\mathrm{SNr}$ during active avoidance?}

The PPT is part of the midbrain locomotor region, which also includes the cuneiform nucleus (Ryczko and Dubuc, 2013; Roseberry et al., 2016; Capelli et al., 2017; Mena-Segovia and Bolam, 2017; Arber and Costa, 2018), and is critically involved in the expression of signaled active avoidance (Hormigo et al., 2019). Optogenetic inhibition of CaMKII-expressing (putative glutamatergic) cells, or excitation of local GABAergic cells, in PPT blocks signaled active avoidance. Moreover, optogenetic excitation of GABAergic cells that project to PPT from $\mathrm{SNr}$ (Hormigo et al., 2019), zona incerta (Hormigo et al., 2020), or striatum (particularly, NAc; Figs. 7,9) also robustly block signaled active avoidance. However, the exact cells and nuclei that form the midbrain locomotor region, or that control signaled active avoidance in PPT, have not been fully defined. We purposefully refer to this area as PPT to distinguish it from the peduculopontine tegmental nucleus proper, which is usually delineated by its cholinergic cell population. The PPT area we have found to control signaled active avoidance is located (in mice) just ventral to the cuneiform area, at $\sim 1.25 \mathrm{~mm}$ lateral from the midline, and $4.7 \mathrm{~mm}$ posterior from bregma. While this corresponds to peduculopontine tegmental nucleus, there are several nuclei in this area, apart from peduculopontine tegmental nucleus, which may be involved in the effects we observed; future work is needed to delineate their contribution. The present results emphasize the significance of PPT in signaled active avoidance because striatotegmental fibers terminated selectively in PPT in the caudal midbrain (specifically delineating a peduncle toward the dorsal pons), and exciting these fibers alone in PPT was sufficient to abolish signaled active avoidance. GABAergic afferents reaching PPT have the ability to suppress signaled active avoidance, indicating a potential permissive role for these afferent nuclei in controlling the expression of the behavior.

SNr cells are not coding the CS and are not driving active avoidance responses. Instead, $\mathrm{SNr}$ cells appear to monitor the movement occurring during avoidance responses. The monitoring may allow regulation of movement speed or other movement variables (Turner and Desmurget, 2010; Panigrahi et al., 2015; Rueda-Orozco and Robbe, 2015; Yttri and Dudman, 2016), in downstream structures, but is not essential to drive the avoidance response. Thus, $\mathrm{SNr}$ firing provides a signal about the occurrence of the movement to downstream structures, which are essential for driving the response. Indeed, we found that inhibition of GABAergic afferents in PPT, including those from SNr and zona incerta, decreased movement speed while not impairing signaled active avoidance, indicating that these afferents regulate avoidance movement in PPT.

While excitation of GABAergic afferents reaching PPT, including those from $\mathrm{SNr}$, can robustly control the expression of active avoidance responses, it is not clear when $\mathrm{SNr}$ excitation occurs naturally to suppress signaled active avoidance responses. There are at least two options. One option is that broad SNr activation sufficient to impair active avoidance responses does not occur normally, and only happens when basal ganglia goes awry during pathology (Bevan et al., 2002; Kravitz et al., 2010; 
Wichmann and Dostrovsky, 2011; Rubin et al., 2012; Willard et al., 2019). Alternatively, broad $\mathrm{SNr}$ excitation may serve to update ongoing responses, such as when these responses must stop immediately because of contingency changes (Schmidt et al., 2013). These possibilities will be explored in future work.

Lesions of the striatum, including the NAc, can facilitate or impair active avoidance (Kirkby and Kimble, 1968; Darvas et al., 2011; Bravo-Rivera et al., 2014; Lichtenberg et al., 2014; Wendler et al., 2014; Ramirez et al., 2015). The striatum appears to be engaged by active avoidance in both humans and rodents (Oleson et al., 2012; Bravo-Rivera et al., 2015; Boeke et al., 2017). Our results show that the striatum and the NAc can control signaled active avoidance responses through a direct pathway to $\mathrm{PPT}$ or indirectly via $\mathrm{SNr}$, and these effects are opposite. Thus, while activity in the direct striatonigral pathway effectively drives avoidance responses by inhibiting $\mathrm{SNr}$, activity in the direct striatotegmental pathway blocks signaled avoidance responses by inhibiting PPT. Further work will have to decipher when these pathways exert their influences.

Since our results highlight a permissive regulatory role, rather than a driving role, for basal ganglia output in the expression of signaled active avoidance behavior, our findings point to other afferents reaching PPT as responsible for driving the CS-related neural activity that drive active avoidance responses.

\section{References}

Albin RL, Young AB, Penney JB (1989) The functional anatomy of basal ganglia disorders. Trends Neurosci 12:366-375.

Arber S, Costa RM (2018) Connecting neuronal circuits for movement. Science 360:1403-1404.

Barbera G, Liang B, Zhang L, Gerfen CR, Culurciello E, Chen R, Li Y, Lin DT (2016) Spatially compact neural clusters in the dorsal striatum encode locomotion relevant information. Neuron 92:202-213.

Barter JW, Li S, Sukharnikova T, Rossi MA, Bartholomew RA, Yin HH (2015) Basal ganglia outputs map instantaneous position coordinates during behavior. J Neurosci 35:2703-2716.

Bevan MD, Magill PJ, Terman D, Bolam JP, Wilson CJ (2002) Move to the rhythm: oscillations in the subthalamic nucleus-external globus pallidus network. Trends Neurosci 25:525-531.

Boeke EA, Moscarello JM, LeDoux JE, Phelps EA, Hartley CA (2017) Active avoidance: neural mechanisms and attenuation of Pavlovian conditioned responding. J Neurosci 37:4808-4818.

Bolles RC (1970) Species-specific defense reactions and avoidance learning. Psychol Rev 77:32-48.

Brandao ML, Cardoso SH, Melo LL, Motta V, Coimbra NC (1994) Neural substrate of defensive behavior in the midbrain tectum. Neurosci Biobehav Rev 18:339-346.

Bravo-Rivera C, Roman-Ortiz C, Brignoni-Perez E, Sotres-Bayon F, Quirk GJ (2014) Neural structures mediating expression and extinction of platform-mediated avoidance. J Neurosci 34:9736-9742.

Bravo-Rivera C, Roman-Ortiz C, Montesinos-Cartagena M, Quirk GJ (2015) Persistent active avoidance correlates with activity in prelimbic cortex and ventral striatum. Front Behav Neurosci 9:184.

Brown J, Pan WX, Dudman JT (2014) The inhibitory microcircuit of the substantia nigra provides feedback gain control of the basal ganglia output. Elife 3:e02397.

Caggiano V, Leiras R, Goni-Erro H, Masini D, Bellardita C, Bouvier J, Caldeira V, Fisone G, Kiehn O (2018) Midbrain circuits that set locomotor speed and gait selection. Nature 553:455-460.

Capelli P, Pivetta C, Soledad Esposito M, Arber S (2017) Locomotor speed control circuits in the caudal brainstem. Nature 551:373-377.

Carelli RM, West MO (1991) Representation of the body by single neurons in the dorsolateral striatum of the awake, unrestrained rat. J Comp Neurol 309:231-249.

Chevalier G, Deniau JM (1990) Disinhibition as a basic process in the expression of striatal functions. Trends Neurosci 13:277-280.
Cohen JD, Castro-Alamancos MA (2007) Early sensory pathways for detection of fearful conditioned stimuli: tectal and thalamic relays. J Neurosci 27:7762-7776.

Cohen JD, Castro-Alamancos MA (2010a) Detection of low salience whisker stimuli requires synergy of tectal and thalamic sensory relays. J Neurosci 30:2245-2256.

Cohen JD, Castro-Alamancos MA (2010b) Neural correlates of active avoidance behavior in superior colliculus. J Neurosci 30:8502-8511.

Cui G, Jun SB, Jin X, Pham MD, Vogel SS, Lovinger DM, Costa RM (2013) Concurrent activation of striatal direct and indirect pathways during action initiation. Nature 494:238-242.

Darvas M, Fadok JP, Palmiter RD (2011) Requirement of dopamine signaling in the amygdala and striatum for learning and maintenance of a conditioned avoidance response. Learn Mem 18:136-143.

DeLong MR (1990) Primate models of movement disorders of basal ganglia origin. Trends Neurosci 13:281-285.

Desmurget M, Turner RS (2008) Testing basal ganglia motor functions through reversible inactivations in the posterior internal globus pallidus. J Neurophysiol 99:1057-1076.

Fan D, Rossi MA, Yin HH (2012) Mechanisms of action selection and timing in substantia nigra neurons. J Neurosci 32:5534-5548.

Freeze BS, Kravitz AV, Hammack N, Berke JD, Kreitzer AC (2013) Control of basal ganglia output by direct and indirect pathway projection neurons. J Neurosci 33:18531-18539.

Gulley JM, Kuwajima M, Mayhill E, Rebec GV (1999) Behavior-related changes in the activity of substantia nigra pars reticulata neurons in freely moving rats. Brain Res 845:68-76.

Hikida T, Kimura K, Wada N, Funabiki K, Nakanishi S (2010) Distinct roles of synaptic transmission in direct and indirect striatal pathways to reward and aversive behavior. Neuron 66:896-907.

Hikosaka O, Wurtz RH (1983) Visual and oculomotor functions of monkey substantia nigra pars reticulata: I. Relation of visual and auditory responses to saccades. J Neurophysiol 49:1230-1253.

Hikosaka O, Takikawa Y, Kawagoe R (2000) Role of the basal ganglia in the control of purposive saccadic eye movements. Physiol Rev 80:953-978.

Hormigo S, Vega-Flores G, Castro-Alamancos MA (2016) Basal ganglia output controls active avoidance behavior. J Neurosci 36:10274-10284.

Hormigo S, Vega-Flores G, Rovira V, Castro-Alamancos MA (2019) Circuits that mediate expression of signaled active avoidance converge in the pedunculopontine tegmentum. J Neurosci 39:4576-4594.

Hormigo S, Zhou J, Castro-Alamancos MA (2020) Zona incerta GABAergic output controls a signaled locomotor action in the midbrain tegmentum. eNeuro 7:ENEURO.0390-19.2020.

Kirkby RJ, Kimble DP (1968) Avoidance and escape behavior following striatal lesions in the rat. Exp Neurol 20:215-227.

Klaus A, Alves da Silva J, Costa RM (2019) What, if, and when to move: basal ganglia circuits and self-paced action initiation. Annu Rev Neurosci 42:459-483.

Kravitz AV, Kreitzer AC (2012) Striatal mechanisms underlying movement, reinforcement, and punishment. Physiology (Bethesda) 27:167-177.

Kravitz AV, Freeze BS, Parker PR, Kay K, Thwin MT, Deisseroth K, Kreitzer AC (2010) Regulation of parkinsonian motor behaviours by optogenetic control of basal ganglia circuitry. Nature 466:622-626.

LeDoux JE, Moscarello J, Sears R, Campese V (2017) The birth, death and resurrection of avoidance: a reconceptualization of a troubled paradigm. Mol Psychiatry 22:24-36.

Lichtenberg NT, Kashtelyan V, Burton AC, Bissonette GB, Roesch MR (2014) Nucleus accumbens core lesions enhance two-way active avoidance. Neuroscience 258:340-346.

Markowitz JE, Gillis WF, Beron CC, Neufeld SQ, Robertson K, Bhagat ND, Peterson RE, Peterson E, Hyun M, Linderman SW, Sabatini BL, Datta SR (2018) The striatum organizes 3D behavior via moment-to-moment action selection. Cell 174:44-58.e17.

Mena-Segovia J, Bolam JP (2017) Rethinking the pedunculopontine nucleus: from cellular organization to function. Neuron 94:7-18.

Mineka S (1979) The role of fear in theories of avoidance learning, flooding, and extinction. Psychol Bull 86:985-1010.

Mink JW (1996) The basal ganglia: focused selection and inhibition of competing motor programs. Prog Neurobiol 50:381-425.

Mowrer OH (1960) Learning theory and behavior. New York: Wiley. 
O’Hare JK, Ade KK, Sukharnikova T, Van Hooser SD, Palmeri ML, Yin HH, Calakos N (2016) Pathway-specific striatal substrates for habitual behavior. Neuron 89:472-479.

Oleson EB, Gentry RN, Chioma VC, Cheer JF (2012) Subsecond dopamine release in the nucleus accumbens predicts conditioned punishment and its successful avoidance. J Neurosci 32:14804-14808.

Pan WX, Brown J, Dudman JT (2013) Neural signals of extinction in the inhibitory microcircuit of the ventral midbrain. Nat Neurosci 16:71-78.

Panigrahi B, Martin KA, Li Y, Graves AR, Vollmer A, Olson L, Mensh BD, Karpova AY, Dudman JT (2015) Dopamine is required for the neural representation and control of movement vigor. Cell 162:1418-1430.

Ramirez F, Moscarello JM, LeDoux JE, Sears RM (2015) Active avoidance requires a serial basal amygdala to nucleus accumbens shell circuit. J Neurosci 35:3470-3477.

Redgrave P, Prescott TJ, Gurney K (1999) The basal ganglia: a vertebrate solution to the selection problem? Neuroscience 89:1009-1023.

Robbe D (2018) To move or to sense? Incorporating somatosensory representation into striatal functions. Curr Opin Neurobiol 52:123-130.

Roseberry TK, Lee AM, Lalive AL, Wilbrecht L, Bonci A, Kreitzer AC (2016) Cell-type-specific control of brainstem locomotor circuits by basal ganglia. Cell 164:526-537.

Rubin JE, McIntyre CC, Turner RS, Wichmann T (2012) Basal ganglia activity patterns in parkinsonism and computational modeling of their downstream effects. Eur J Neurosci 36:2213-2228.

Rueda-Orozco PE, Robbe D (2015) The striatum multiplexes contextual and kinematic information to constrain motor habits execution. Nat Neurosci 18:453-460.

Ryczko D, Dubuc R (2013) The multifunctional mesencephalic locomotor region. Curr Pharm Des 19:4448-4470.

Sales-Carbonell C, Taouali W, Khalki L, Pasquet MO, Petit LF, Moreau T, Rueda-Orozco PE, Robbe D (2018) No discrete start/stop signals in the dorsal striatum of mice performing a learned action. Curr Biol 28:30443055.e3045.

Schmidt R, Leventhal DK, Mallet N, Chen F, Berke JD (2013) Canceling actions involves a race between basal ganglia pathways. Nat Neurosci $16: 1118-1124$

Schultz W (1998) Predictive reward signal of dopamine neurons. J Neurophysiol 80:1-27.

Shang C, Liu Z, Chen Z, Shi Y, Wang Q, Liu S, Li D, Cao P (2015) A parvalbumin-positive excitatory visual pathway to trigger fear responses in mice. Science 348:1472-1477.
Smith Y, Bevan MD, Shink E, Bolam JP (1998) Microcircuitry of the direct and indirect pathways of the basal ganglia. Neuroscience 86:353-387.

Tai LH, Lee AM, Benavidez N, Bonci A, Wilbrecht L (2012) Transient stimulation of distinct subpopulations of striatal neurons mimics changes in action value. Nat Neurosci 15:1281-1289.

Tecuapetla F, Matias S, Dugue GP, Mainen ZF, Costa RM (2014) Balanced activity in basal ganglia projection pathways is critical for contraversive movements. Nat Commun 5:4315.

Tepper JM, Martin LP, Anderson DR (1995) GABAA receptor-mediated inhibition of rat substantia nigra dopaminergic neurons by pars reticulata projection neurons. J Neurosci 15:3092-3103.

Turner RS, Desmurget M (2010) Basal ganglia contributions to motor control: a vigorous tutor. Curr Opin Neurobiol 20:704-716.

Wendler E, Gaspar JC, Ferreira TL, Barbiero JK, Andreatini R, Vital MA, Blaha CD, Winn P, Da Cunha C (2014) The roles of the nucleus accumbens core, dorsomedial striatum, and dorsolateral striatum in learning: performance and extinction of Pavlovian fear-conditioned responses and instrumental avoidance responses. Neurobiol Learn Mem 109:27-36.

Wichmann T, Dostrovsky JO (2011) Pathological basal ganglia activity in movement disorders. Neuroscience 198:232-244.

Wiegert JS, Oertner TG (2016) How (not) to silence long-range projections with light. Nat Neurosci 19:527-528.

Wiegert JS, Mahn M, Prigge M, Printz Y, Yizhar O (2017) Silencing neurons: tools, applications, and experimental constraints. Neuron 95:504-529.

Willard AM, Isett BR, Whalen TC, Mastro KJ, Ki CS, Mao X, Gittis AH (2019) State transitions in the substantia nigra reticulata predict the onset of motor deficits in models of progressive dopamine depletion in mice. Elife 8:e42746

Wurtz RH, Hikosaka O (1986) Role of the basal ganglia in the initiation of saccadic eye movements. Prog Brain Res 64:175-190.

Yin HH (2017) The basal ganglia in action. Neuroscientist 23:299-313.

Yin HH, Knowlton BJ (2006) The role of the basal ganglia in habit formation. Nat Rev Neurosci 7:464-476.

Yin HH, Mulcare SP, Hilario MR, Clouse E, Holloway T, Davis MI, Hansson AC, Lovinger DM, Costa RM (2009) Dynamic reorganization of striatal circuits during the acquisition and consolidation of a skill. Nat Neurosci 12:333-341.

Yttri EA, Dudman JT (2016) Opponent and bidirectional control of movement velocity in the basal ganglia. Nature 533:402-406. 